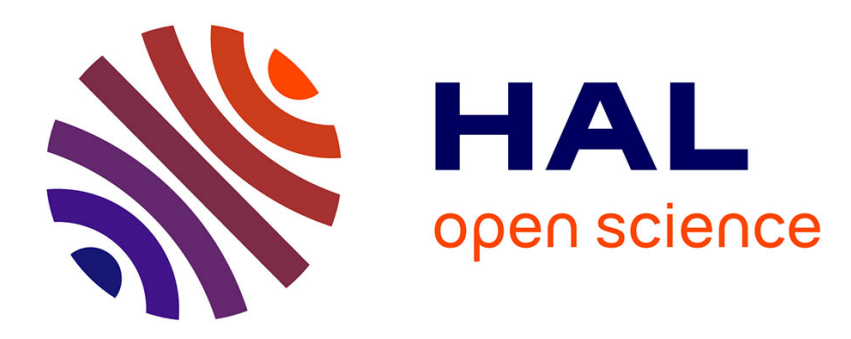

\title{
Introduction to random fields and scale invariance
} Hermine Biermé

\section{To cite this version:}

Hermine Biermé. Introduction to random fields and scale invariance. 2017. hal-01493834v2

\section{HAL Id: hal-01493834 \\ https://hal.science/hal-01493834v2}

Preprint submitted on 5 May 2018

HAL is a multi-disciplinary open access archive for the deposit and dissemination of scientific research documents, whether they are published or not. The documents may come from teaching and research institutions in France or abroad, or from public or private research centers.
L'archive ouverte pluridisciplinaire HAL, est destinée au dépôt et à la diffusion de documents scientifiques de niveau recherche, publiés ou non, émanant des établissements d'enseignement et de recherche français ou étrangers, des laboratoires publics ou privés. 


\title{
Introduction to random fields and scale invariance
}

\author{
Hermine Biermé
}

\begin{abstract}
In medical imaging, several authors have proposed to characterize roughness of observed textures by their fractal dimensions. Fractal analysis of 1D signals is mainly based on the stochastic modeling using the famous fractional Brownian motion for which the fractal dimension is determined by its so-called Hurst parameter. Lots of $2 \mathrm{D}$ generalizations of this toy model may be defined according to the scope. This lecture intends to present some of them. After an introduction to random fields, the first part will focus on the construction of Gaussian random fields with prescribed invariance properties such as stationarity, self-similarity, or operator scaling property. Sample paths properties such as modulus of continuity and Hausdorff dimension of graphs will be settled in the second part to understand links with fractal analysis. The third part will concern some methods of simulation and estimation for these random fields in a discrete setting. Some applications in medical imaging will be presented. Finally, the last part will be devoted to geometric constructions involving Marked Poisson Point Processes and shot noise processes.
\end{abstract}

\section{Random fields and scale invariance}

We recall in this section definitions and properties of random fields. Most of them can also be found in [22] but we try here to detail some important proofs. We stress on invariance properties such as stationarity, isotropy, and scale invariance and illustrate these properties with typical examples.

Hermine Biermé

LMA, UMR CNRS 7348, Université de Poitiers, bd Marie et Pierre Curie, 86962 Chasseneuil, France, e-mail: hermine.bierme@math.univ-poitiers.fr 


\subsection{Introduction to random fields}

As usual when talking about randomness, we let $(\Omega, \mathscr{A}, \mathbb{P})$ be a probability space, reflecting variability.

\subsubsection{Definitions and distribution}

Let us first recall the general definition of a stochastic process. For this purpose we have to consider a set of indices $T$. In this lecture we assume that $T \subset \mathbb{R}^{d}$ for some dimension $d \geq 1$.

Definition 1. A (real) stochastic process indexed by $T$ is just a collection of real random variables meaning that for all $t \in T$, one has $X_{t}:(\Omega, \mathscr{A}) \rightarrow(\mathbb{R}, \mathscr{B}(\mathbb{R}))$ measurable.

Stochastic processes are very important in stochastic modeling as they can mimic numerous natural phenomena. For instance, when $d=1$, one can choose $T \subset \mathbb{R}$ (seen as time parameters) and consider $X_{t}(\omega)$ as the real value of heart frequency at time $t \in T$ with noise measurement or for an individual $\omega \in \Omega$. Note that, in practice data are only available on a discrete finite subset $S$ of $T$, for instance each millisecond. When $d=2$, choosing $T=[0,1]^{2}$, the value $X_{t}(\omega)$ may correspond to the grey level of a picture at point $t \in T$. Again, in practice, data are only available on pixels $S=\{0,1 / n, \ldots, 1\}^{2} \subset T$ for an image of size $(n+1) \times(n+1)$. In general we talk about random fields when $d>1$ and keep the terminology stochastic process only for $d=1$. Since we have actually a map $X$ from $\Omega \times T$ with values in $\mathbb{R}$ we can also consider it as a map from $\Omega$ to $\mathbb{R}^{T}$. We equip $\mathbb{R}^{T}$ with the smallest $\sigma$-algebra $\mathscr{C}$ such that the projections $\pi_{t}:\left(\mathbb{R}^{T}, \mathscr{C}\right) \rightarrow(\mathbb{R}, \mathscr{B}(\mathbb{R}))$, defined by $\pi_{t}(f)=f(t)$ are measurable. It follows that $X:(\Omega, \mathscr{A}) \rightarrow\left(\mathbb{R}^{T}, \mathscr{C}\right)$ is measurable and its distribution is defined as the image measure of $\mathbb{P}$ by $X$, which is a probability measure on $\left(\mathbb{R}^{T}, \mathscr{C}\right)$. An important consequence of Kolmogorov's consistency theorem (see [37] p.92) is the following equivalent definition.

Definition 2. The distribution of $\left(X_{t}\right)_{t \in T}$ is given by all its finite dimensional distribution (fdd) ie the distribution of all real random vectors

$$
\left(X_{t_{1}}, \ldots, X_{t_{k}}\right) \text { for } k \geq 1, t_{1}, \ldots, t_{k} \in T
$$

Note that joint distributions for random vectors of arbitrary size $k$ are often difficult to compute. However we can infer some statistics of order one and two by considering only couples of variables.

Definition 3. The stochastic process $\left(X_{t}\right)_{t \in T}$ is a second order process if $\mathbb{E}\left(X_{t}^{2}\right)<+\infty$, for all $t \in T$. In this case we define

- its mean function $m_{X}: t \in T \rightarrow \mathbb{E}\left(X_{t}\right) \in \mathbb{R}$;

- its covariance function $K_{X}:(t, s) \in T \times T \rightarrow \operatorname{Cov}\left(X_{t}, X_{s}\right) \in \mathbb{R}$. 
A particular case arises when $m_{X}=0$ and the process $X$ is said centered. Otherwise the stochastic process $Y=X-m_{X}$ is also second order and now centered with the same covariance function $K_{Y}=K_{X}$. Hence we will mainly consider centered stochastic processes. The covariance function of a stochastic process must verify the following properties.

Proposition 1. A function $K: T \times T \rightarrow \mathbb{R}$ is a covariance function iff

1. $K$ is symmetric ie $K(t, s)=K(s, t)$ for all $(t, s) \in T \times T$;

2. $K$ is non-negative definite: $\forall k \geq 1, t_{1}, \ldots, t_{k} \in T,: \lambda_{1}, \ldots, \lambda_{k} \in \mathbb{R}$,

$$
\sum_{i, j=1}^{k} \lambda_{i} \lambda_{j} K\left(t_{i}, t_{j}\right) \geq 0
$$

Proof. The first implication is trivial once remarked the fact that $\operatorname{Var}\left(\sum_{i=1}^{k} \lambda_{i} X_{t_{i}}\right)=$ $\sum_{i, j=1}^{k} \lambda_{i} \lambda_{j} K\left(t_{i}, t_{j}\right)$. For the converse, we need to introduce Gaussian processes.

\subsubsection{Gaussian processes}

As far as second order properties are concerned the most natural class of processes are given by Gaussian ones.

Definition 4. A stochastic process $\left(X_{t}\right)_{t \in T}$ is a Gaussian process if for all $k \geq 1$ and $t_{1}, \ldots, t_{k} \in T$

$$
\left(X_{t_{1}}, \ldots, X_{t_{k}}\right) \text { is a Gaussian vector of } \mathbb{R}^{k},
$$

which is equivalent to the fact that for all $\lambda_{1}, \ldots, \lambda_{k} \in \mathbb{R}$, the real random variable $\sum_{i=1}^{k} \lambda_{i} X_{t_{i}}$ is a Gaussian variable (eventually degenerate ie constant).

Note that this definition completely characterizes the distribution of the process in view of Definition 2.

Proposition 2. When $\left(X_{t}\right)_{t \in T}$ is a Gaussian process, $\left(X_{t}\right)_{t \in T}$ is a second order process and its distribution is determined by its mean function $m_{X}: t \mapsto \mathbb{E}\left(X_{t}\right)$ and its covariance function $K_{X}:(t, s) \mapsto \operatorname{Cov}\left(X_{t}, X_{s}\right)$.

This comes from the fact that the distribution of the Gaussian vector $\left(X_{t_{1}}, \ldots, X_{t_{k}}\right)$ is characterized by its mean $\left(\mathbb{E}\left(X_{t_{1}}\right), \ldots, \mathbb{E}\left(X_{t_{k}}\right)\right)=\left(m_{X}\left(t_{1}\right), \ldots, m_{X}\left(t_{k}\right)\right)$ and its covariance matrix $\left(\operatorname{Cov}\left(X_{t_{i}}, X_{t_{j}}\right)\right)_{1 \leq i, j \leq k}=\left(K_{X}\left(t_{i}, t_{j}\right)\right)_{1 \leq i, j \leq k}$.

Again Kolmogorov's consistency theorem (see [37] p.92 for instance) allows to prove the following existence result that finishes to prove Proposition 1.

Theorem 1. Let $m: T \rightarrow \mathbb{R}$ and $K: T \times T \rightarrow \mathbb{R}$ a symmetric and non-negative definite function, then there exists a Gaussian process with mean $m$ and covariance $K$. 
Let us give some insights of construction for the fundamental example of Gaussian process, namely the Brownian motion. We set here $T=\mathbb{R}^{+}$and consider $\left(X_{k}\right)_{k \in \mathbb{N}}$ a family of independent identically distributed second order random variables with $\mathbb{E}\left(X_{k}\right)=0$ and $\operatorname{Var}\left(X_{k}\right)=1$. For any $n \geq 1$, we construct on $T$ the following stochastic process

$$
S_{n}(t)=\frac{1}{\sqrt{n}} \sum_{k=1}^{[n t]} X_{k}
$$

By the central limit theorem (see [28] for instance) we clearly have for $t>0$, $\sqrt{\frac{n}{[n t]}} S_{n}(t) \underset{n \rightarrow+\infty}{\stackrel{d}{\longrightarrow}} \mathscr{N}(0,1)$ so that by Slutsky's theorem (see [15] for instance) $S_{n}(t) \underset{n \rightarrow+\infty}{\stackrel{d}{\longrightarrow}} \mathscr{N}(0, t)$. Moreover, for $k \geq 1$, if $0<t_{1}<\ldots<t_{k}$, by independence of marginals,

$$
\left(S_{n}\left(t_{1}\right), S_{n}\left(t_{2}\right)-S_{n}\left(t_{1}\right), \ldots, S_{n}\left(t_{k}\right)-S_{n}\left(t_{k-1}\right)\right) \underset{n \rightarrow+\infty}{\stackrel{d}{\longrightarrow}} Z=\left(Z_{1}, \ldots, Z_{k}\right),
$$

with $Z \sim \mathscr{N}\left(0, K_{Z}\right)$ for $K_{Z}=\operatorname{diag}\left(t_{1}, t_{2}-t_{1}, \ldots, t_{k}-t_{k-1}\right)$. Hence identifying the $k \times k$ matrix $P_{k}=\left(\begin{array}{cccc}1 & 0 & \ldots & 0 \\ 1 & 1 & \\ \vdots & & \ddots & \\ 1 & \ldots & \ldots & 1\end{array}\right)$ with the corresponding linear application on $\mathbb{R}^{k}$,

$$
\begin{aligned}
\left(S_{n}\left(t_{1}\right), S_{n}\left(t_{2}\right), \ldots, S_{n}\left(t_{k}\right)\right) & \underset{n \rightarrow+\infty}{\stackrel{d}{\longrightarrow}} P_{k} Z,
\end{aligned}
$$

with $P_{k} Z \sim \mathscr{N}\left(0, P_{k} K_{Z} P_{k}^{*}\right)$ and $P_{k} K_{Z} P_{k}^{*}=\left(\min \left(t_{i}, t_{j}\right)\right)_{1 \leq i, j \leq k}$. In particular the function

$$
K(t, s)=\min (t, s)=\frac{1}{2}(t+s-|t-s|)
$$

is a covariance function on the whole space $\mathbb{R}^{+} \times \mathbb{R}^{+}$and $\left(S_{n}\right)_{n}$ converges in finite dimensional distribution to a centered Gaussian stochastic process $X=\left(X_{t}\right)_{t \in \mathbb{R}^{+}}$ with covariance $K$, known (up to continuity of sample paths) as the standard Brownian motion on $\mathbb{R}^{+}$.

Fig. 1 Sample paths of a Brownian motion on $[0,1]$. The realization is obtained using fast and exact synthesis presented in Section 3.1.1

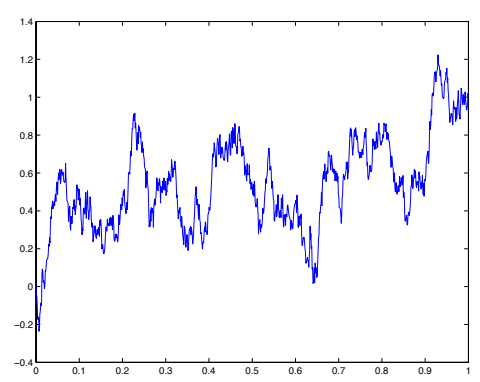


Now we can extend this process on $\mathbb{R}$ by simply considering $X^{(1)}$ and $X^{(2)}$ two independent centered Gaussian processes on $\mathbb{R}^{+}$with covariance function $K$ and defining $B_{t}:=X_{t}^{(1)}$ for $t \geq 0, B_{t}:=X_{-t}^{(2)}$ for $t<0$. Computing the covariance function of $B$ yields the following definition.

Definition 5. A (standard) Brownian motion on $\mathbb{R}$ is a centered Gaussian process $\left(B_{t}\right)_{t \in \mathbb{R}}$ with covariance function given by

$$
K_{B}(t, s)=\operatorname{Cov}\left(B_{t}, B_{s}\right)=\frac{1}{2}(|t|+|s|-|t-s|), \forall t, s \in \mathbb{R} .
$$

From Gaussian stochastic processes defined on $\mathbb{R}$ we can define Gaussian random fields defined on $\mathbb{R}^{d}$ in several ways. We give some possibilities in the next section.

\subsubsection{Gaussian fields defined from processes}

We consider on $\mathbb{R}^{d}$ the Euclidean norm, denoted by $\|\cdot\|$ with respect to the Euclidean scalar product $x \cdot y$ for $x, y \in \mathbb{R}^{d}$. The unit sphere $\left\{\theta \in \mathbb{R}^{d} ;\|\theta\|=1\right\}$ is denoted as $S^{d-1}$ and we let $\left(e_{i}\right)_{1 \leq i \leq d}$ stand for the canonical basis of $\mathbb{R}^{d}$.

A first example of construction is given in the following proposition.

Proposition 3. Let $K: \mathbb{R} \times \mathbb{R} \rightarrow \mathbb{R}$ be a continuous covariance function. For all $\mu$ non-negative finite measure on the unit sphere $S^{d-1}$, the function defined by

$$
(x, y) \in \mathbb{R}^{d} \times \mathbb{R}^{d} \mapsto \int_{S^{d-1}} K(x \cdot \theta, y \cdot \theta) d \mu(\theta) \in \mathbb{R},
$$

is a covariance function on $\mathbb{R}^{d} \times \mathbb{R}^{d}$.

Proof. According to Proposition 1, it is enough to check symmetry and nonnegative definiteness. Symmetry is clear and for all $k \geq 1, x_{1}, \ldots, x_{k} \in \mathbb{R}^{d}, \lambda_{1}, \ldots, \lambda_{k} \in$ $\mathbb{R}$,

$$
\sum_{i, j=1}^{k} \lambda_{i} \lambda_{j} \int_{S^{d-1}} K\left(x_{i} \cdot \theta, x_{j} \cdot \theta\right) d \mu(\theta)=\int_{S^{d-1}}\left(\sum_{i, j=1}^{k} \lambda_{i} \lambda_{j} K\left(x_{i} \cdot \theta, x_{j} \cdot \theta\right)\right) d \mu(\theta) \geq 0
$$

since for all $\theta \in S^{d-1}, x_{1} \cdot \theta, \ldots, x_{k} \cdot \theta \in \mathbb{R}$ with $K$ non-negative definite on $\mathbb{R} \times \mathbb{R}$ and $\mu$ non-negative measure.

As an example we can note that $\int_{S^{d-1}}|x \cdot \theta| d \theta=c_{d}\|x\|$, with $c_{d}=\int_{S^{d-1}}\left|e_{1} \cdot \theta\right| d \theta$ for $e_{1}=(1,0, \ldots, 0) \in S^{d-1}$. Then, for $K_{B}$ the covariance function of a standard Brownian motion on $\mathbb{R}$ we get

$$
\int_{S^{d-1}} K_{B}(x \cdot \theta, y \cdot \theta) d \theta=\frac{c_{d}}{2}(\|x\|+\|y\|-\|x-y\|) .
$$


Definition 6. A (standard) Lévy Chentsov field on $\mathbb{R}^{d}$ is a centered Gaussian field $\left(X_{x}\right)_{x \in \mathbb{R}^{d}}$ with covariance function given by

$$
\operatorname{Cov}\left(X_{x}, X_{y}\right)=\frac{1}{2}(\|x\|+\|y\|-\|x-y\|), \forall x, y \in \mathbb{R}^{d} .
$$

Let us note that $\left(X_{t \theta}\right)_{t \in \mathbb{R}}$ is therefore a standard Brownian motion for all $\theta \in S^{d-1}$.
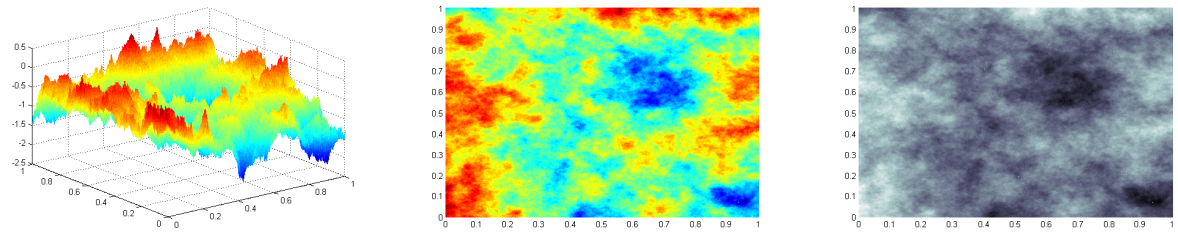

Fig. 2 Sample paths of a Lévy Chentsov field on $[0,1]^{2}$. The realization is obtained using fast and exact synthesis presented in Section 3.1.3. On the left we draw the obtained surface, on the right the two corresponding images with colors or gray levels given according to the values on each points.

Another example is given using a sheet structure according to the following proposition.

Proposition 4. Let $K_{1}, K_{2}, \ldots, K_{d}$ be covariance functions on $\mathbb{R} \times \mathbb{R}$, then the function defined by

$$
(x, y) \in \mathbb{R}^{d} \times \mathbb{R}^{d} \mapsto \prod_{i=1}^{d} K_{i}\left(x \cdot e_{i}, y \cdot e_{i}\right) \in \mathbb{R}
$$

is a covariance function on $\mathbb{R}^{d} \times \mathbb{R}^{d}$.

Proof. Since for $1 \leq i \leq d$, the function $K_{i}$ is a covariance function on $\mathbb{R} \times \mathbb{R}$ we may consider independent centered Gaussian processes $X^{(i)}=\left(X_{t}^{(i)}\right)_{t \in \mathbb{R}}$ with covariance given by $K_{i}$. For $x=\left(x_{1}, \ldots, x_{d}\right) \in \mathbb{R}^{d}$, we may define the random variable $X_{x}=\prod_{i=1}^{d} X_{x \cdot e_{i}}^{(i)}$ so that the random field $X=\left(X_{x}\right)_{x \in \mathbb{R}^{d}}$ is second order (but no more Gaussian!), centered, with covariance given by

$$
\operatorname{Cov}\left(X_{x}, X_{y}\right)=\mathbb{E}\left(X_{x} X_{y}\right)=\prod_{i=1}^{d} \mathbb{E}\left(X_{x \cdot e_{i}}^{(i)} X_{y \cdot e_{i}}^{(i)}\right)=\prod_{i=1}^{d} K_{i}\left(x_{i}, y_{i}\right),
$$

by independence of $X^{(1)}, \ldots, X^{(d)}$.

This leads to the second fundamental extension of Brownian motion on the whole space $\mathbb{R}^{d}$, by choosing $K_{i}=K_{B}$ for all $1 \leq i \leq d$.

Definition 7. A (standard) Brownian sheet on $\mathbb{R}^{d}$ is a centered Gaussian field $\left(X_{x}\right)_{x \in \mathbb{R}^{d}}$ with covariance function given by 


$$
\operatorname{Cov}\left(X_{x}, X_{y}\right)=\prod_{i=1}^{d} \frac{1}{2}\left(\left|x \cdot e_{i}\right|+\left|y \cdot e_{i}\right|-\left|x \cdot e_{i}-y \cdot e_{i}\right|\right), \forall x, y \in \mathbb{R}^{d}
$$

Note that it implies that this field is equal to 0 on the axes $\left\{x \in \mathbb{R}^{d} ; \exists i \in\{1, \ldots, d\}, x\right.$. $\left.e_{i}=0\right\}$ and corresponds to a Brownian motion (non-standard) when restricted to $\left\{x+t e_{i} ; t \in \mathbb{R}\right\}$, for $x \in \mathbb{R}^{d}$ with $x \cdot e_{i}=0$. The following section will focus on some invariance properties.

\subsection{Stationarity and Invariances}

When considering stochastic modeling of homogeneous media it is usual to assume an invariance of distributions under translation (stationarity) or vectorial rotation (isotropy).

\subsubsection{Stationarity and Isotropy}

Definition 8. The random field $X=\left(X_{x}\right)_{x \in \mathbb{R}^{d}}$ is (strongly) stationary if, for all $x_{0} \in$ $\mathbb{R}^{d}$, the random field $\left(X_{x+x_{0}}\right)_{x \in \mathbb{R}^{d}}$ has the same distribution than $X$.

It implies a specific structure of second order moments.

Proposition 5. If $X=\left(X_{x}\right)_{x \in \mathbb{R}^{d}}$ is a stationary second order random field, then,

- its mean function is constant $m_{X}(x)=m_{X}$, for all $x \in \mathbb{R}^{d}$ and some $m_{X} \in \mathbb{R}$;

- its covariance may be written as $K_{X}(x, y)=c_{X}(x-y)$ with $c_{X}: \mathbb{R}^{d} \rightarrow \mathbb{R}$ an even function satisfying

(i) $c_{X}(0) \geq 0$;

(ii) $\left|c_{X}(x)\right| \leq c_{X}(0) \forall x \in \mathbb{R}^{d}$;

(iii) $c_{X}$ is of non-negative type ie $\forall k \geq 1, x_{1}, \ldots, x_{k} \in \mathbb{R}^{d}, \lambda_{1}, \ldots, \lambda_{k} \in \mathbb{C}$,

$$
\sum_{j, l=1}^{k} \lambda_{j} \bar{\lambda}_{l} c_{X}\left(x_{j}-x_{l}\right) \geq 0
$$

Let us remark that the two above properties characterize the weak (second-order) stationarity. Note also that they imply strong stationarity when the field is assumed to be Gaussian. This is because mean and covariance functions characterize the distribution of Gaussian fields.

Proof. Since $X_{x} \stackrel{d}{=} X_{0}$ we get $m_{X}(x)=\mathbb{E}\left(X_{x}\right)=\mathbb{E}\left(X_{0}\right):=m_{X}$. For the covariance structure we set $c_{X}(z)=K_{X}(z, 0)$, for all $z \in \mathbb{R}^{d}$, and remark that for $y \in \mathbb{R}^{d}$, one has $\left(X_{z+y}, X_{y}\right) \stackrel{d}{=}\left(X_{z}, X_{0}\right)$ so that $\operatorname{Cov}\left(X_{z+y}, X_{y}\right)=c_{X}(z)$. Hence for $z=x-y$ we obtain $K_{X}(x, y)=c_{X}(x-y)$. Since $\left(X_{x}, X_{0}\right) \stackrel{d}{=}\left(X_{0}, X_{-x}\right)$, the function $c_{X}$ is even. The first point comes from the fact that $c_{X}(0)=\operatorname{Var}\left(X_{0}\right)=\operatorname{Var}\left(X_{x}\right) \geq 0$, the second one is 
obtained using Cauchy-Schwarz inequality to bound $\left|\operatorname{Cov}\left(X_{x}, X_{0}\right)\right|$. The last one is just a reformulation of the non-negative definiteness of $K_{X}$ when $\lambda_{1}, \ldots, \lambda_{k} \in \mathbb{R}$. Otherwise, it follows writing $\lambda_{j}=a_{j}+i b_{j}$ since we have $\Re\left(\lambda_{j} \overline{\lambda_{l}}\right)=a_{j} a_{l}+b_{j} b_{l}$ and $\mathfrak{I}\left(\lambda_{j} \overline{\lambda_{l}}\right)=b_{j} a_{l}-b_{l} a_{j}$ with $c_{X}$ even.

Remark that when a function $c: \mathbb{R}^{d} \rightarrow \mathbb{R}$ satisfies (1), then $c$ must be even and satisfy points $(i)$ and $(i i)$. Actually, $(i)$ is obtained for $k=1, \lambda_{1}=1$ and $x_{1}=0$. Considering $k=2, x_{1}=0$ and $x_{2}=x \in \mathbb{R}^{d}$, we first obtain for $\lambda_{1}=1$ and $\lambda_{2}=i$ that $2 c(0)+i c(x)-i c(-x) \geq 0$ hence $c$ is even, while for $\lambda_{1}=\lambda_{2}=1$ it yields $-c(x) \leq c(0)$ and for $\lambda_{1}=1=-\lambda_{2}$ we get $c(x) \leq c(0)$ so that $(i i)$ is satisfied.

By Bochner's theorem (1932), a continuous function of non-negative type is a Fourier transform of a non-negative finite measure. This can be rephrased as the following theorem.

Theorem 2 (Bochner). A continuous function $c: \mathbb{R}^{d} \rightarrow \mathbb{R}$ is of non-negative type if and only if $c(0) \geq 0$ and there exists a symmetric probability measure $v$ on $\mathbb{R}^{d}$ such that

$$
\forall x \in \mathbb{R}^{d}, c(x)=c(0) \int_{\mathbb{R}^{d}} e^{i x \cdot \xi} d v(\xi) .
$$

In other words there exists a symmetric random vector $Z$ on $\mathbb{R}^{d}$ such that

$$
\forall x \in \mathbb{R}^{d}, c(x)=c(0) \mathbb{E}\left(e^{i x \cdot Z}\right) .
$$

When $c=c_{X}$ is the covariance of a random field $X$, the measure $v=v_{X}$ is called the spectral measure of $X$. This strong result implies in particular that we may define stationary centered Gaussian random field with a covariance function given by the characteristic function of a symmetric random vector.

Proof. Note that the converse implication is straightforward so we will only prove the first one. We may assume that $c(0)>0$, otherwise there is nothing to prove. The first step is to assume that $c \in L^{1}\left(\mathbb{R}^{d}\right)$. Note that in view of (ii), since $c$ is bounded we also have $c \in L^{2}\left(\mathbb{R}^{d}\right)$. We will prove that its Fourier transform $\hat{c} \in L^{2}\left(\mathbb{R}^{d}\right)$ is necessarily non-negative. To this end remark that, approximating by Riemann sums for instance, we necessarily have

$$
\int_{\mathbb{R}^{d}} \int_{\mathbb{R}^{d}} g(x) \overline{g(y)} c(x-y) d x d y \geq 0,
$$

for all $g \in \mathscr{S}\left(\mathbb{R}^{d}\right)$, the Schwartz class of infinitely differentiable function with rapid decreasing. We denote as usual $\hat{g}(\xi)=\int_{\mathbb{R}^{d}} e^{-i x \cdot \xi} g(x) d x$, the Fourier transform, that may be extended to any $L^{2}\left(\mathbb{R}^{d}\right)$ function. We may rewrite $\int_{\mathbb{R}^{d}} \int_{\mathbb{R}^{d}} g(x) \overline{g(y)} c(x-$ $y) d x d y=\int_{\mathbb{R}^{d}} g(x) c * \bar{g}(x) d x$, where $*$ is the usual convolution product on $L^{1}\left(\mathbb{R}^{d}\right)$ and $c * \bar{g} \in L^{2}\left(\mathbb{R}^{d}\right)$ since $c \in L^{1}\left(\mathbb{R}^{d}\right)$ and $g \in \mathscr{S}\left(\mathbb{R}^{d}\right) \subset L^{2}\left(\mathbb{R}^{d}\right)$. Hence, by Plancherel's theorem (see [55] for instance), we have

$$
\frac{1}{(2 \pi)^{d}} \int_{\mathbb{R}^{d}} \hat{c}(\xi)|\hat{g}(-\xi)|^{2} d \xi=\int_{\mathbb{R}^{d}} g(x) c * \bar{g}(x) d x,
$$


where the right hand side is non-negative in view of (2). Now, for $\sigma>0$, let us denote by $h_{\sigma}$ the density of a centered Gaussian vector of $\mathbb{R}^{d}$ with covariance $\sigma^{2} I_{d}$ ie $h_{\sigma}(x)=\frac{1}{\sigma^{d}(2 \pi)^{d / 2}} e^{-\frac{\|x\|^{2}}{2 \sigma^{2}}}$. Its characteristic function is given by the Fourier transform $\hat{h}_{\sigma}(\xi)=e^{-\sigma^{2} \frac{\|\xi\|^{2}}{2}}$. In this way $\left(h_{\sigma}\right)_{\sigma}$ is an approximation of identity and $c * h_{\sigma}(x) \rightarrow$ $c(x)$, as $\sigma \rightarrow 0$, since $c$ is continuous. Moreover, $\hat{c}$ is also continuous as the Fourier transform of an $L^{1}\left(\mathbb{R}^{d}\right)$ function, so that we also have $\hat{c} * h_{\sigma}(\xi) \rightarrow \hat{c}(\xi)$. Now we will prove that $\hat{c} \geq 0$. Let us take $g_{\sigma}(x)=\frac{\sigma^{d / 2}}{2^{d / 4} \pi^{3 d / 4}} e^{-\sigma^{2}\|x\|^{2}}$ such that $\left|\hat{g}_{\sigma}\right|^{2}=h_{\sigma}$, by (2) and (3), we obtain that for all $\sigma>0$

$$
\hat{c} * h_{\sigma}(0)=\int_{\mathbb{R}^{d}} \hat{c}(\xi) h_{\sigma}(\xi) d \xi=(2 \pi)^{d} \int_{\mathbb{R}^{d}} g_{\sigma}(x) c * \overline{g_{\sigma}}(x) d x \geq 0 .
$$

Letting $\sigma$ tend to 0 we get $\hat{c}(0) \geq 0$. But for all $\xi \in \mathbb{R}^{d}$, the function $e^{-i \xi} c$ is an $L^{1}\left(\mathbb{R}^{d}\right)$ function satisfying (1). Hence its Fourier transform is non-negative at point 0 , according to previously. But this is exactly $\hat{c}(\xi)$ and therefore $\hat{c}$ is nonnegative. Using Fatou Lemma in (1), for $g=h_{\sigma}$ as $\sigma$ tends to 0 we also obtain that $\int_{\mathbb{R}^{d}} \hat{c}(\xi) d \xi \leq c(0)$ ensuring that $\hat{c} \in L^{1}\left(\mathbb{R}^{d}\right)$. Then, by the Fourier inversion theorem, since $c$ and $\hat{c}$ are even, we get

$$
c(x)=\frac{1}{(2 \pi)^{d}} \int_{\mathbb{R}^{d}} e^{i x \cdot \xi} \hat{c}(\xi) d \xi,
$$

with $c(0)=\frac{1}{(2 \pi)^{d}} \int_{\mathbb{R}^{d}} \hat{c}(\xi) d \xi$. Hence we can choose $Z$ a random vector with density given by $\hat{c} /\left((2 \pi)^{d} c(0)\right)$.

For the general case we remark that $c \hat{h}_{\sigma}$ is also a function of non-negative type. Actually, since $\hat{h}_{\sigma}$ is the Fourier transform of a non-negative function, by converse of Bochner's theorem it is of non-negative type and we may consider $X_{\sigma}$ a centered stationary Gaussian random field with covariance $\hat{h}_{\sigma}$. Let us also consider $X$ a centered stationary Gaussian random field with covariance $c$, independent from $X_{\sigma}$. Then the random field $X X_{\sigma}$ is stationary and admits $c \hat{h}_{\sigma}$ for covariance function. Since $c$ is bounded by $(i i)$, the function $c \hat{h}_{\sigma}$ is in $L^{1}\left(\mathbb{R}^{d}\right)$ and we may find $Z_{\sigma}$ such that $\left[c \hat{h}_{\sigma}\right]=c(0) \mathbb{E}\left(e^{i x \cdot Z_{\sigma}}\right)$. But $c \hat{h}_{\sigma}$ tends to $c$ which is continuous at 0 as $\sigma$ tends to 0 . Hence, by Lévy's theorem (see [28] for instance), there exists a random vector $Z$ such that $Z_{\sigma} \underset{\sigma \rightarrow 0}{\stackrel{d}{\longrightarrow}} Z$ and $v=\mathbb{P}_{Z}$ is convenient. Let us finally conclude that $Z$ is symmetric since $c$ is even.

Examples of stationary Gaussian processes are given by Ornstein Uhlenbeck processes constructed on $\mathbb{R}$ with a parameter $\theta>0$ and $B$ a standard Brownian motion on $\mathbb{R}^{+}$, by

$$
X_{t}=e^{-\theta t} B_{e^{2 \theta t}}, \forall t \in \mathbb{R}
$$

Then $X=\left(X_{t}\right)_{t}$ is clearly a centered Gaussian process with covariance

$$
\operatorname{Cov}\left(X_{t}, X_{s}\right)=e^{-\theta|t-s|}:=c_{X}(t-s), \forall t, s \in \mathbb{R} .
$$




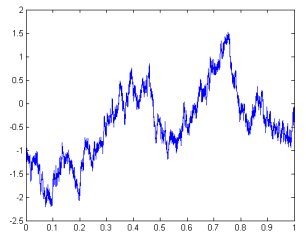

$\theta=5$

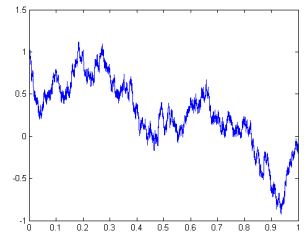

$\theta=1$

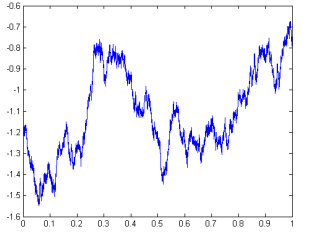

$\theta=1 / 5$

Fig. 3 Sample paths of Ornstein Uhlenbeck process on $[0,1]$, using fast and exact synthesis via circulant embedding matrix method.

Hence it is weakly stationary and also strongly since it is Gaussian. Now the spectral measure is given by $v_{X}(d t)=\frac{\theta^{2}}{\pi\left(\theta^{2}+t^{2}\right)} d t$, or equivalently $c_{X}(t)=\mathbb{E}\left(e^{i t \cdot Z_{\theta}}\right)$, with $Z_{\theta}$ a random variable with Cauchy distribution of parameter $\theta$.

Definition 9. The random field $X=\left(X_{x}\right)_{x \in \mathbb{R}^{d}}$ is isotropic if, for all $R$ rotation of $\mathbb{R}^{d}$, the random field $\left(X_{R x}\right)_{x \in \mathbb{R}^{d}}$ has the same distribution than $X$.

Note that, contrarily to the stationarity, the notion of isotropy is useless in dimension 1 ! We already have seen one example of isotropic random field when considering the Lévy Chentsov random field. Actually, for all $R$ rotation of $\mathbb{R}^{d}$,

$$
\operatorname{Cov}\left(X_{R x}, X_{R y}\right)=\frac{1}{2}(\|R x\|+\|R y\|-\|R x-R y\|)=\operatorname{Cov}\left(X_{x}, X_{y}\right) .
$$

Since $X$ is centered and Gaussian this implies that $\left(X_{R x}\right)_{x \in \mathbb{R}^{d}}$ has the same distribution than $X$. However $X$ is not stationary (note that $X(0)=0$ a.s.). An example of stationary and isotropic random field may be given by considering Gaussian covariances $\hat{h}_{\sigma}$, for $\sigma>0$ (with $Z_{\sigma} \sim \mathscr{N}\left(0, \sigma I_{d}\right)$ in Bochner's theorem). Let us also remark that considering the covariance function $k_{\sigma}(t, s)=e^{-\sigma^{2}(t-s) / 2}$ on $\mathbb{R} \times \mathbb{R}$ we also have

$$
K_{\sigma}(x, y)=\hat{h}_{\sigma}(x-y)=\prod_{i=1}^{d} k_{\sigma}\left(x \cdot e_{i}, y \cdot e_{i}\right),
$$

so that this field has also a sheet structure as in Proposition 4. Since $\hat{h}_{\sigma}$ is isotropic, we also have $K_{\sigma}(R x, R y)=K_{\sigma}(x, y)$ for $x, y \in \mathbb{R}^{d}$, and this allows to define a stationary isotropic centered Gaussian field with covariance $K_{\sigma}$.

Another very important invariance property is the scale invariance also called self-similarity for random fields.

\subsubsection{Self-similarity or scale invariance}

Definition 10. The random field $X=\left(X_{x}\right)_{x \in \mathbb{R}^{d}}$ is self-similar of order $H>0$ if, for all $\lambda>0$, the random field $\left(X_{\lambda x}\right)_{x \in \mathbb{R}^{d}}$ has the same distribution than $\lambda^{H} X=$ $\left(\lambda^{H} X_{x}\right)_{x \in \mathbb{R}^{d}}$. 


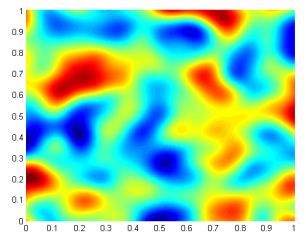

$\sigma^{2}=100$

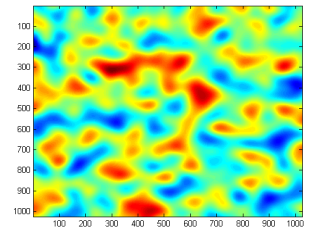

$\sigma^{2}=300$

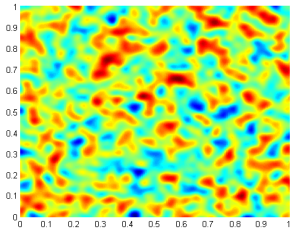

$\sigma^{2}=1000$

Fig. 4 Sample paths of center Gaussian random fields with Gaussian covariances on $[0,1]^{2}$, obtained using circulant embedding matrix method (see [53] for details).

Note that the Lévy Chentsov field, and in particular the Brownian motion $(d=1)$, is self-similar of order $H=1 / 2$ since

$$
\begin{aligned}
\operatorname{Cov}\left(X_{\lambda x}, X_{\lambda y}\right) & =\frac{1}{2}(\|\lambda x\|+\|\lambda y\|-\|\lambda x-\lambda y\|) \\
& =\lambda \operatorname{Cov}\left(X_{x}, X_{y}\right)=\operatorname{Cov}\left(\lambda^{1 / 2} X_{x}, \lambda^{1 / 2} X_{y}\right)
\end{aligned}
$$

Recall that $X$ is isotropic but not stationary. Actually, there does not exist a nontrivial stationary self-similar second order field since we should have $\operatorname{Var}\left(X_{x}\right)=$ $\operatorname{Var}\left(X_{\lambda x}\right)=\lambda^{2 H} \operatorname{Var}\left(X_{x}\right)$ for all $\lambda>0$ and $x \in \mathbb{R}^{d}$, implying that $\operatorname{Var}\left(X_{x}\right)=0$. In order to define self-similar fields for homogeneous media we must relax stationary property. This is done throught the notion of stationary increments.

\subsubsection{Stationary increments}

Definition 11. The random field $X=\left(X_{x}\right)_{x \in \mathbb{R}^{d}}$ has (strongly) stationary increments if, for all $x_{0} \in \mathbb{R}^{d}$, the random field $\left(X_{x+x_{0}}-X_{x_{0}}\right)_{x \in \mathbb{R}^{d}}$ has the same distribution than $\left(X_{x}-X_{0}\right)_{x \in \mathbb{R}^{d}}$.

Of course a stationary random field $X$ has stationary increments but this class is larger: it also contents $X-X_{0}$ for instance that can not be stationary except if it is almost surely equal to 0 . An example of field with stationary increments is given by the Levy Chentsov field $X$ since we have

$$
\operatorname{Cov}\left(X_{x+x_{0}}-X_{x_{0}}, X_{y+x_{0}}-X_{x_{0}}\right)=\operatorname{Cov}\left(X_{x}-X_{0}, X_{y}-X_{0}\right),
$$

using the fact that $X_{0}=0$ a.s. We have an analogous of Proposition 5 concerning second order structure of fields with stationary increments.

Proposition 6. If $X=\left(X_{x}\right)_{x \in \mathbb{R}^{d}}$ is a second order centered random field with stationary increments and $X_{0}=0$ a.s., then its covariance function may be written as

$$
K_{X}(x, y)=\frac{1}{2}\left(v_{X}(x)+v_{X}(y)-v_{X}(x-y)\right)
$$


with the function $v_{X}(x)=\operatorname{Var}\left(X_{x+x_{0}}-X_{x_{0}}\right)=\operatorname{Var}\left(X_{x}-X_{0}\right)=\operatorname{Var}\left(X_{x}\right)$ called variogram satisfying

1. $v_{X}(0)=0$

2. $v_{X}(x) \geq 0$ and $v_{X}(-x)=v_{X}(x)$

3. $v_{X}$ is conditionally of negative type ie $\forall k \geq 1, x_{1}, \ldots, x_{k} \in \mathbb{R}^{d}, \lambda_{1}, \ldots, \lambda_{k} \in \mathbb{C}$,

$$
\sum_{j=1}^{k} \lambda_{j}=0 \Rightarrow \sum_{j, l=1}^{k} \lambda_{j} \overline{\lambda_{l}} v_{X}\left(x_{j}-x_{l}\right) \leq 0
$$

Note that when $X_{0}$ does not vanish a.s. this proposition applies to $X-X_{0}$.

Proof. Compute $\operatorname{Var}\left(X_{x}-X_{y}\right)=\operatorname{Var}\left(X_{x}\right)+\operatorname{Var}\left(X_{x}\right)+2 K_{X}(x, y)$ and note that $X_{x}-$ $X_{y} \stackrel{d}{=} X_{x-y}-X_{0}=X_{x-y}$ to get $K_{X}$ with respect to $v_{X}$. We clearly have $v_{X} \geq 0$ as a variance and $v_{X}(0)=0$ since $X_{0}=0$ a.s. The evenness comes from $X_{-x}=X_{-x}-$ $X_{0} \stackrel{d}{=} X_{0}-X_{x}=-X_{x}$. The last property follows from the fact that

$$
\operatorname{Var}\left(\sum_{j=1}^{k} \lambda_{j} X_{x_{j}}\right)=\frac{1}{2} \sum_{j, l=1}^{k} \lambda_{j} \lambda_{l}\left(v_{X}\left(x_{j}\right)+v_{X}\left(x_{l}\right)-v_{X}\left(x_{j}-x_{l}\right)\right) \geq 0,
$$

for $\lambda_{1}, \ldots, \lambda_{k} \in \mathbb{R}$, using the expression of $K_{X}\left(x_{j}, x_{l}\right)$ with respect to $v_{X}$. The inequality is extended for $\lambda_{1}, \ldots, \lambda_{k} \in \mathbb{C}$ as in Proposition 5 since $v_{X}$ is also even.

In order to define centered Gaussian random fields we can use the following result.

Theorem 3 (Schoenberg). Let $v: \mathbb{R}^{d} \rightarrow \mathbb{R}$ be a function such that $v(0)=0$. The following are equivalent.

i) $v$ is conditionally of negative type;

ii) $K:(x, y) \in \mathbb{R}^{d} \times \mathbb{R}^{d} \mapsto \frac{1}{2}(v(x)+v(y)-v(x-y))$ is a covariance function;

iii)For all $\lambda>0$, the function $e^{-\lambda v}$ is of non-negative type.

Proof. To prove that $i) \Rightarrow i i$ ), we use Proposition 1 . Symmetry comes from the fact that $v$ is even. Actually, taking $k=2, \lambda_{1}=i=-\lambda_{2}$ and $x_{1}=x, x_{2}=0$ we obtain that $v(x) \leq v(-x)$ since $v(0)=0$, such that replacing $x$ by $-x$ we get $v(x)=v(-x)$. For the second point let $k \geq 1, x_{1}, \ldots, x_{k} \in \mathbb{R}^{d}, \lambda_{1}, \ldots, \lambda_{k} \in \mathbb{R}$ and set $\lambda_{0}=-\sum_{i=1}^{k} \lambda_{i}$ and $x_{0}=0$. We compute $\sum_{i, j=1}^{k} \lambda_{i} \lambda_{j} K\left(x_{i}, x_{j}\right)$ as

$$
\begin{aligned}
\sum_{i, j=1}^{k} \lambda_{i} \lambda_{j} v\left(x_{i}\right)-\frac{1}{2} \sum_{i, j=1}^{k} \lambda_{i} \lambda_{j} v\left(x_{i}-x_{j}\right) & =-\lambda_{0} \sum_{i=1}^{k} \lambda_{i} v\left(x_{i}\right)-\frac{1}{2} \sum_{j=1}^{k} \lambda_{j} \sum_{i=1}^{k} \lambda_{i} v\left(x_{i}-x_{j}\right) \\
& =-\frac{1}{2} \lambda_{0} \sum_{i=1}^{k} \lambda_{i} v\left(x_{i}\right)-\frac{1}{2} \sum_{j=0}^{k} \lambda_{j} \sum_{i=1}^{k} \lambda_{i} v\left(x_{i}-x_{j}\right) \\
& =-\frac{1}{2} \sum_{j=0}^{k} \lambda_{j} \sum_{i=0}^{k} \lambda_{i} v\left(x_{i}-x_{j}\right) \geq 0,
\end{aligned}
$$


since $v$ is even and conditionally of negative type.

Let us now consider $i i) \Rightarrow i i i)$. Let $\left(X^{(n)}\right)_{n}$ be a sequence of iid centered Gaussian random fields with covariance function given by $K$ and $N$ an independent Poisson random variable of parameter $\lambda>0$. We may define a new random field $Y=\prod_{n=1}^{N} X^{(n)}$, with the convention that $\prod_{n=1}^{0}=1$. Therefore, for all $x, y \in \mathbb{R}^{d}$, we get

$$
\begin{aligned}
\mathbb{E}\left(Y_{x} Y_{y}\right) & =\sum_{k=0}^{+\infty} \mathbb{E}\left(Y_{x} Y_{y} \mid N=k\right) \mathbb{P}(N=k) \\
& =\sum_{k=0}^{+\infty} \mathbb{E}\left(\prod_{n=1}^{k} X_{x}^{(n)} X_{y}^{(n)}\right) e^{-\lambda} \frac{\lambda^{k}}{k !} \\
& =\sum_{k=0}^{+\infty} K(x, y)^{k} e^{-\lambda} \frac{\lambda^{k}}{k !}=e^{-\lambda(1-K(x, y))},
\end{aligned}
$$

by independence. Now remark that $\mathbb{E}\left(Y_{x}^{2}\right)=e^{-\lambda(1-K(x, x))}=e^{-\lambda(1-v(x))}$. Hence defining the random field $Z$ by setting $Z_{x}=\frac{Y_{x}}{\sqrt{\mathbb{E}\left(Y_{x}^{2}\right)}} e^{\lambda / 2}$ we get

$$
\mathbb{E}\left(Z_{x} Z_{y}\right)=\frac{e^{\lambda}}{e^{-\lambda\left(1-\frac{1}{2}(v(x)+v(y))\right)}} e^{-\lambda(1-K(x, y))}=e^{-\frac{\lambda}{2} v(x-y)} .
$$

As a consequence, for all $k \geq 1, x_{1}, \ldots, x_{k} \in \mathbb{R}^{d}$ and $\lambda_{1}, \ldots, \lambda_{k} \in \mathbb{R}$

$$
\sum_{j, l}^{k} \lambda_{j} \lambda_{l} e^{-\frac{\lambda}{2} v\left(x_{j}-x_{l}\right)}=\mathbb{E}\left(\left(\sum_{j}^{k} \lambda_{j} Z_{x_{j}}\right)^{2}\right) \geq 0 .
$$

Note that $v$ must be even since $K(x, 0)=K(0, x)$ by symmetry of a covariance function and $v(0)=0$ so that the previous inequality extends to $\lambda_{1}, \ldots, \lambda_{k} \in \mathbb{C}$. This finishes to prove iii).

The last implication iii) $\Rightarrow i$ ) comes from the fact for $k \geq 1, \lambda_{1}, \ldots, \lambda_{k} \in \mathbb{C}$ s.t. $\sum_{j=1}^{k} \lambda_{j}=0, x_{1}, \ldots, x_{k} \in \mathbb{R}^{d}$, we may write for all $\varepsilon>0$,

$$
\sum_{j, l=1}^{k} \lambda_{j} \bar{\lambda}_{l} v\left(x_{j}-x_{l}\right) \frac{1}{\varepsilon} \int_{0}^{\varepsilon} e^{-\lambda v\left(x_{j}-x_{l}\right)} d \lambda=\frac{1}{\varepsilon} \sum_{j, l=1}^{k} \lambda_{j} \overline{\lambda_{l}}\left(1-e^{-\varepsilon v\left(x_{j}-x_{l}\right)}\right) \geq 0,
$$

since $\sum_{j=1}^{k} \lambda_{j}=0$ and $e^{-\varepsilon v}$ is of non-negative type. Hence letting $\varepsilon$ tend to 0 we get the result.

As an application of this result we may deduce that the function $v(x)=\|x\|^{2}$ is a variogram. Actually, this easily follows from bi-linearity of the Euclidean product since $v(x-y)=\|x\|^{2}+\|y\|^{2}-2 x \cdot y$. But we can also remark that for all $\sigma>0$, the function $e^{-\frac{\sigma^{2}}{2} v}$ is the Gaussian covariance function which implies that $v$ is conditionally of negative type. Let us remark that this variogram corresponds to a kind 
of trivial field since choosing $Z \sim \mathscr{N}\left(0, I_{d}\right)$, one can define the centered Gaussian random field $X_{x}=x \cdot Z$, for $x \in \mathbb{R}^{d}$, that admits $v$ for variogram.

An important corollary for the construction of self-similar fields with stationary increments is the following one due to J. Istas in [35].

Corollary 1. If $v: \mathbb{R}^{d} \rightarrow \mathbb{R}^{+}$is a variogram then the function $v^{H}$ is also a variogram for all $H \in(0,1]$.

Proof. There is nothing to prove for $H=1$ and when $H \in(0,1)$, it is sufficient to remark that, by a change of variable, one has for $t \geq 0$,

$$
t^{H}=c_{H} \int_{0}^{+\infty} \frac{1-e^{-\lambda t}}{\lambda^{H+1}} d \lambda
$$

for $c_{H}^{-1}=\int_{0}^{+\infty} \frac{1-e^{-\lambda}}{\lambda^{H+1}} d \lambda \in(0,+\infty)$. Hence, for $k \geq 1, \lambda_{1}, \ldots, \lambda_{k} \in \mathbb{C}$ s.t. $\sum_{j=1}^{k} \lambda_{j}=0$, $x_{1}, \ldots, x_{k} \in \mathbb{R}^{d}$, we get

$$
\sum_{j, l=1}^{k} \lambda_{j} \bar{\lambda}_{l} v\left(x_{j}-x_{l}\right)^{H}=-c_{H} \int_{0}^{+\infty} \sum_{j, l=1}^{k} \lambda_{j} \bar{\lambda}_{l} e^{-\lambda v\left(x_{j}-x_{l}\right)} \lambda^{-H-1} d \lambda \leq 0,
$$

in view of Schoenberg's theorem since $v$ is a variogram.

It follows that for all $H \in(0,1]$ the function $v_{H}(x)=\|x\|^{2 H}$ is conditionally of negative type and leads to the next definition.

Definition 12. A (standard) fractional Brownian field on $\mathbb{R}^{d}$, with Hurst parameter $H \in(0,1]$, is a centered Gaussian field $\left(B_{H}\right)_{x \in \mathbb{R}^{d}}$ with covariance function given by

$$
\operatorname{Cov}\left(B_{H}(x), B_{H}(y)\right)=\frac{1}{2}\left(\|x\|^{2 H}+\|y\|^{2 H}-\|x-y\|^{2 H}\right), \forall x, y \in \mathbb{R}^{d} .
$$

Of course when $H=1 / 2$, we recognize $B_{1 / 2}$ as the Lévy Chentsov field. The order of self-similarity is now given by $H$. Note also that the case $H=1$ corresponds to a degenerate case where $B_{1}=(x \cdot Z)_{x}$ for $Z \sim \mathscr{N}\left(0, I_{d}\right)$.

Proposition 7. Up to a constant, the fractional Brownian field of order $H \in(0,1]$ is the unique isotropic centered Gaussian field with stationary increments which is self-similar of order $H$.

Proof. This comes from the fact that the distribution of a centered Gaussian field $X$ with stationary increments is characterized by its variogram $v_{X}$ as soon as $X(0)=$ 0 a.s. Self-similarity implies that $X(0)=0$ and therefore, for all $\lambda>0$ we have $v_{X}(\lambda x)=\operatorname{Var}\left(X_{\lambda x}\right)=\operatorname{Var}\left(\lambda^{H} X_{x}\right)=\lambda^{2 H} \operatorname{Var}\left(X_{x}\right)=\lambda^{2 H} v_{X}(x)$. Hence for all $x \neq 0$, $v_{X}(x)=\|x\|^{2 H} v_{X}\left(\frac{x}{\|x\|}\right)$. But isotropy also implies that $X_{\theta} \stackrel{d}{=} X_{e_{1}}$ for all $\theta \in S^{d-1}$ and $e_{1}=(1,0, \ldots, 0) \in S^{d-1}$. Hence $v_{X}(\theta)=v_{X}\left(e_{1}\right)$ and $X \stackrel{d}{=} \sqrt{v_{X}\left(e_{1}\right)} B_{H}$.

Let us also remark that we cannot find a second order field with stationary increments that is self-similar for an order $H>1$. Actually, by triangular inequality for $\|\cdot\|_{2}:=\sqrt{\operatorname{Var}(\cdot)}$, when $X$ is a second order field, we have $\left\|X_{2 x}\right\|_{2} \leq \| X_{2 x}-$ 


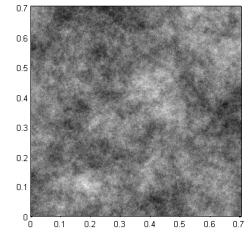

$H=0.2$

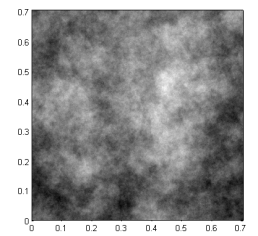

$H=0.4$

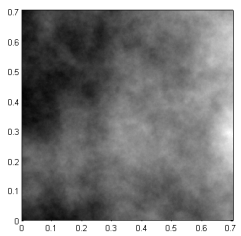

$H=0.6$

Fig. 5 Sample paths realizations of fractional Brownian fields on $[0,1]^{2}$ for different values of $H$, obtained using the fast and exact synthesis method presented in Section 3.1.3.

$X_{x}\left\|_{2}+\right\| X_{x} \|_{2}$, for all $x \in \mathbb{R}^{d}$. Self-similarity of order $H>0$ implies that $X_{0}=0$ a.s. and $\left\|X_{2 x}\right\|_{2}=2^{H}\left\|X_{x}\right\|_{2}$, while stationary increments imply that $\left\|X_{2 x}-X_{x}\right\|_{2}=$ $\left\|X_{x}-X_{0}\right\|_{2}=\left\|X_{x}\right\|_{2}$. Therefore we must have $2^{H-1}\left\|X_{x}\right\|_{2} \leq 1$ and $H \leq 1$ or $X_{x}=0$ for all $x \in \mathbb{R}^{d}$.

In dimension $d=1$, it is called fractional Brownian motion, implicitly introduced in [41] and defined in [47]. The order of self-similarity $H$ is also called Hurst parameter. Hence the fractional Brownian field is an isotropic generalization of this process. Other constructions using sheet structure are known as fractional Brownian sheets but these fields loose stationary increments (see e.g. [22]).

Anisotropy may be an interesting property in applications (see [16] for instance). We also refer to [2] for many examples of anisotropic variograms. For instance, we can consider several anisotropic generalizations of fractional Brownian motions by keeping self-similarity and stationary increments properties following Proposition 3. Let $H \in(0,1)$ and $v_{H}: t \in \mathbb{R} \mapsto|t|^{2 H}$ be the variogram of a fractional Brownian motion that is conditionally of negative type. If $\mu$ is a finite positive measure on $S^{d-1}$, we may define on $\mathbb{R}^{d}$,

$$
v_{H, \mu}(x)=\int_{S^{d-1}} v_{H}(x \cdot \theta) \mu(d \theta)=\int_{S^{d-1}}|x \cdot \theta|^{2 H} \mu(d \theta)=c_{H, \mu}\left(\frac{x}{\|x\|}\right)\|x\|^{2 H},
$$

that is now a conditionally of negative type function on $\mathbb{R}^{d}$. Hence we may consider $X_{H, \mu}=\left(X_{H, \mu}(x)\right)_{x \in \mathbb{R}^{d}}$ a centered Gaussian random field with stationary increments and variogram given by $v_{H, \mu}$. This new random field is still self-similar of order $H$ but may not be isotropic according to the choice of $\mu$. The function $c_{H, \mu}$, describing anisotropy, is called topothesy function as in [25].

For instance, when $d=2$ we can choose $\mu(d \theta)=\mathbf{1}_{(-\alpha, \alpha)}(\theta) d \theta$ for some $\alpha \in(0, \pi / 2]$. Let $\beta_{H}(t)=\int_{0}^{t} u^{H-1 / 2}(1-u)^{H-1 / 2} d u, t \in[0,1]$, be a Beta incomplete function. Then the corresponding topothesy function denoted now by $c_{H, \alpha}$ is a $\pi$ periodic function defined on $(-\pi / 2, \pi / 2]$ by 


$$
c_{H, \alpha}(\theta)=2^{2 H}\left\{\begin{array}{cc}
\beta_{H}\left(\frac{1-\sin (\alpha-\theta)}{2}\right)+\beta_{H}\left(\frac{1+\sin (\alpha+\theta)}{2}\right) & \text { if }-\alpha \leq \theta+\frac{\pi}{2} \leq \alpha \\
\beta_{H}\left(\frac{1+\sin (\alpha-\theta)}{2}\right)+\beta_{H}\left(\frac{1-\sin (\alpha+\theta)}{2}\right) & \text { if }-\alpha \leq \theta-\frac{\pi}{2} \leq \alpha \\
\left|\beta_{H}\left(\frac{1-\sin (\alpha-\theta)}{2}\right)-\beta_{H}\left(\frac{1+\sin (\alpha+\theta)}{2}\right)\right| & \text { otherwise }
\end{array}\right.
$$

We refer to [13] for computations details and to Figure 6 for plots of these functions.
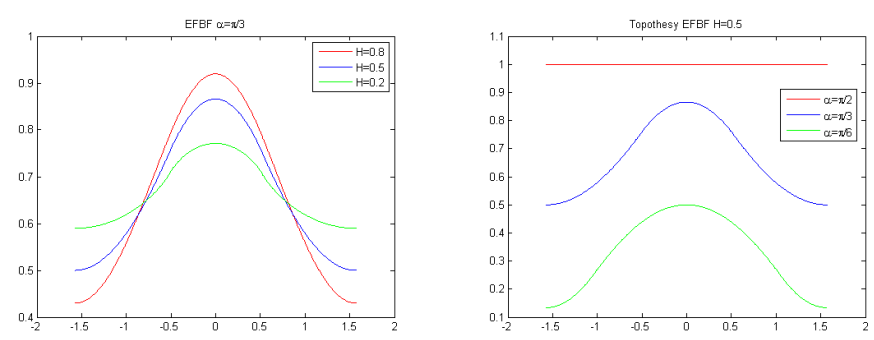

Fig. 6 Topothesy functions of some elementary anisotropic fractional Brownian fields

The associated Gaussian random field denoted by $X_{H, \alpha}$ is called elementary anisotropic fractional Brownian field. Several realizations are presented in Figure 7 for different values of parameters $\alpha$ and $H$. Note that when $\alpha=\pi / 2$ the random field $X_{H, \alpha}$ is isotropic and therefore corresponds to a non-standard fractional Brownian field.

Another anisotropic generalization is obtained by considering a generalization of the self-similarity property that allows different scaling behavior according to directions.

\subsubsection{Operator scaling Property}

Definition 13. Let $E$ be a real $d \times d$ matrix with eigenvalues of positive real parts and $H>0$. The random field $X=\left(X_{x}\right)_{x \in \mathbb{R}^{d}}$ is $(E, H)$-operator scaling if for all $\lambda>0$, the random field $\left(X_{\lambda E_{x}}\right)_{x \in \mathbb{R}^{d}}$ has the same distribution than $\lambda^{H} X=\left(\lambda^{H} X_{x}\right)_{x \in \mathbb{R}^{d}}$, where $\lambda^{E}=\exp (E \log \lambda)$ with $\exp (A)=\sum_{k=0}^{\infty} \frac{A^{k}}{k !}$ the matrix exponential.

Note that when $E$ is the identity matrix we recover the definition of self-similarity of order $H$. Note also that $(E, H)$-operator scaling property is equivalent to $(E / H, 1)$ operator scaling property. We refer to [12] for general cases and focus here on a simple example where the matrix $E$ is assumed to be diagonalizable. In particular when $E$ is a diagonal matrix, this property may be observed for limit of aggregated discrete random fields (see $[42,52]$ for instance). In the general case where $E$ is diagonalizable, we assume that its eigenvalues, denoted by $\alpha_{1}^{-1}, \ldots, \alpha_{d}^{-1}$, are all greater than 1 . They are also eigenvalues of the transpose matrix $E^{t}$ and we denote 


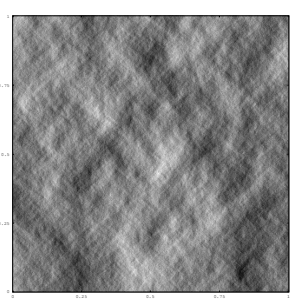

$H=0.2, \alpha=\pi / 3$

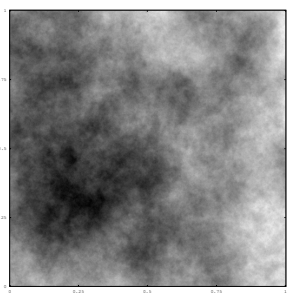

$\alpha=\pi / 2, H=0.5$

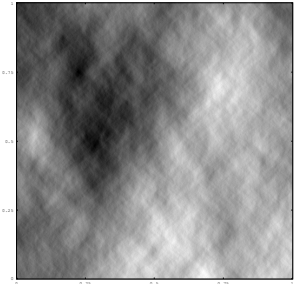

$H=0.5, \alpha=\pi / 3$

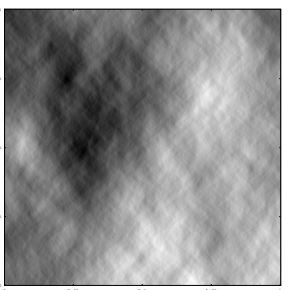

$\alpha=\pi / 3, H=0.5$

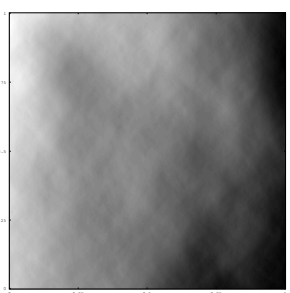

$H=0.8, \alpha=\pi / 3$

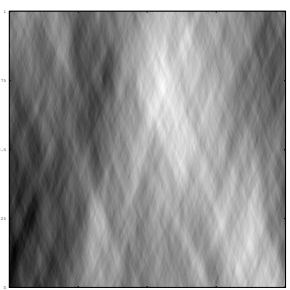

$\alpha=\pi / 6, H=0.5$

Fig. 7 Sample paths realizations of some elementary anisotropic fractional Brownian fields on $[0,1]^{2}$ using Turning band method presented in Section 3.1.2 (see also [13])

by $\theta_{1}, \ldots, \theta_{d}$ the corresponding eigenvectors such that $E^{t} \theta_{i}=\alpha_{i}^{-1} \theta_{i}$. We obtained in [11] the following proposition.

Proposition 8. For $H \in(0,1]$, the function defined on $\mathbb{R}^{d}$ by

$$
v_{H, E}(x)=\tau_{E}(x)^{2 H}=\left(\sum_{i=1}^{d}\left|x \cdot \theta_{i}\right|^{2 \alpha_{i}}\right)^{H}=\left(\sum_{i=1}^{d} v_{\alpha_{i}}\left(x \cdot \theta_{i}\right)\right)^{H}
$$

is a variogram.

Proof. According to Corollary 1, it is enough to prove that $\tau_{E}(x)^{2}$ is a variogram but this follows from the fact that $v_{\alpha_{i}}\left(x \cdot \theta_{i}\right)$ is the variogram of $\left(B_{\alpha_{i}}^{(i)}\left(x \cdot \theta_{i}\right)\right)_{x \in \mathbb{R}^{d}}$, where $B_{\alpha_{1}}^{(1)}, \ldots, B_{\alpha_{d}}^{(d)}$ are $d$ independent fractional Brownian motions on $\mathbb{R}$ with Hurst parameter given by $\alpha_{1}, \ldots, \alpha_{d} \in(0,1]$.

Therefore, we can consider a centered Gaussian random field $X_{H, E}=\left(X_{H, E}(x)\right)_{x \in \mathbb{R}^{d}}$ with stationary increments and variogram given by $v_{H, E}$. Then, $X_{H, E}$ is $(E, H)$ operator scaling since $v_{H, E}\left(\lambda^{E} x\right)=\lambda^{2 H} v_{H, E}(x)$ for all $x \in \mathbb{R}^{d}$. When $\alpha_{1}=\ldots=$ $\alpha_{d}=\alpha \in(0,1]$, or equivalently when $E=\alpha^{-1} I_{d}$, the random field $X_{H, E}$ is actually $\alpha H \in(0,1)$ self-similar with topothesy function given by $v_{H, E}\left(\frac{x}{\|x\|}\right)$. Now considering $\left(\theta_{i}\right)_{1 \leq i \leq d}=\left(e_{i}\right)_{1 \leq i \leq d}$, the canonical basis, such fields are examples 
of Minkowski fractional Brownian fields (see Proposition 3.3 in [49]). In particular, when $d=2$, we get the topothesy functions as the $\pi$-periodic functions $\theta \mapsto\left(|\cos (\theta)|^{2 \alpha}+|\sin (\theta)|^{2 \alpha}\right)^{H}$ (see Figure 8 for some plots).
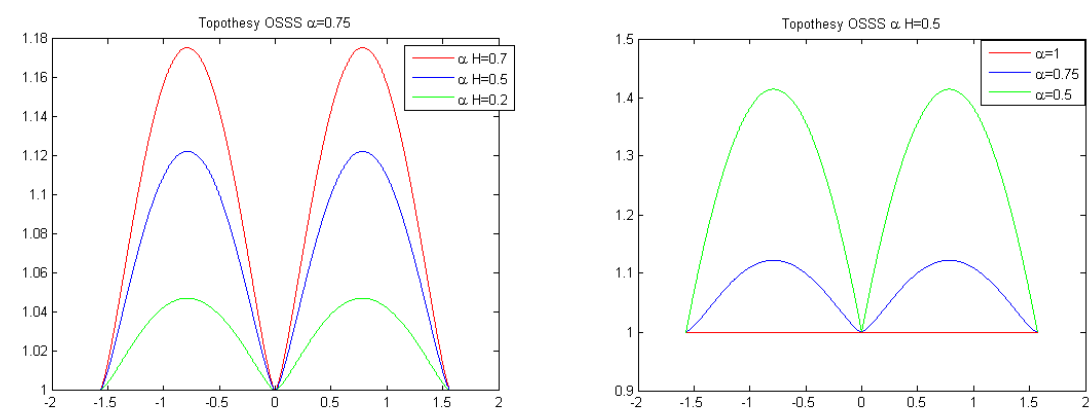

Fig. 8 Topothesy functions of self-similar fields $X_{H, E}$ obtained for $E=\alpha^{-1} I_{d}$.

Some realizations of operator scaling self-similar random fields are presented in Figure 9. Note that the case where $\alpha=1$ corresponds to the isotropic case, whereas the case where $H=1$ is a degenerate one obtained by adding two independent fractional Brownian processes.

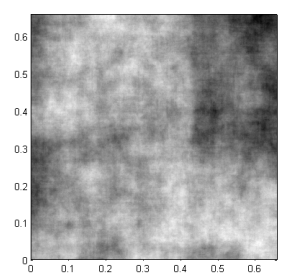

$H=0.6$

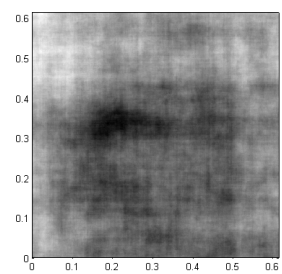

$H=0.7$

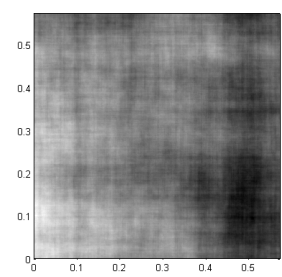

$H=0.8$

Fig. 9 Sample paths realizations of self-similar fields $X_{H, E}$ obtained for $E=\alpha^{-1} I_{d}$ and $H \alpha=0.5$, using the simulation method presented in Section 3.1.3.

The realizations of Figure 10 are no more self-similar but when restricting to horizontal, respectively vertical, lines we get fractional Brownian motions of order $H \alpha_{1}$, respectively $H \alpha_{2}$. The sheet structure appearing as $H$ increases to 1 should come from the reduction of dependency between directions. The vertical gradient comes from the fact that the self-similarity exponent $H \alpha_{2}$ is chosen greater than the horizontal one. This is also linked to sample paths regularity as we will see in the following section. 


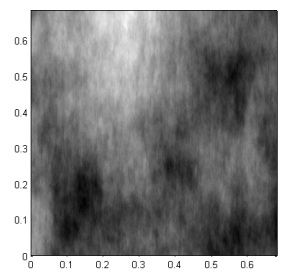

$H=0.6$

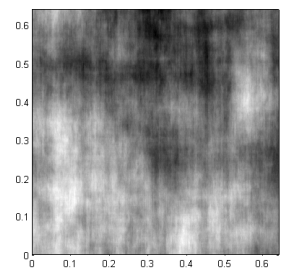

$H=0.7$

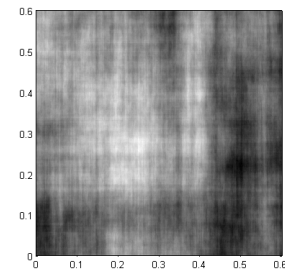

$H=0.8$

Fig. 10 Sample paths realizations of operator scaling fields $X_{H, E}$ obtained for $E=\operatorname{diag}\left(\alpha_{1}^{-1}, \alpha_{2}^{-1}\right)$ with $H \alpha_{1}=0.5$ and $H \alpha_{2}=0.6$, using the simulation method presented in Section 3.1.3.

\section{Sample paths properties}

In this section we focus on Hölder sample paths properties related to fractal properties of Gaussian random fields. In particular we will see that self-similarity properties induce such fractal behaviors.

\subsection{Sample paths regularity}

Before considering sample paths continuity we must introduce the weak notion of stochastic continuity.

Definition 14. Let $X=\left(X_{x}\right)_{x \in \mathbb{R}^{d}}$ be a random field. We say that $X$ is stochastically continuous at point $x_{0} \in \mathbb{R}^{d}$ if

$$
\forall \varepsilon>0, \lim _{x \rightarrow x_{0}} \mathbb{P}\left(\left|X_{x}-X_{x_{0}}\right|>\varepsilon\right)=0 .
$$

Let us emphasize that a centered Gaussian random field with stationary increments is stochastically continuous as soon as its variogram is continuous at point 0 , according to Bienaymé Chebyshev's inequality. Since we have only defined random fields as a collection of real variables $\left(X_{x}\right)_{x \in \mathbb{R}^{d}}$, when studying the functions $x \mapsto X_{x}(\omega)$ for some typical $\omega \in \Omega$, we can in general only state results for a modification of $X$.

Definition 15. Let $X=\left(X_{x}\right)_{x \in \mathbb{R}^{d}}$ be a random field. We say that $\tilde{X}=\left(\tilde{X}_{x}\right)_{x \in \mathbb{R}^{d}}$ is a modification of $X$ if

$$
\forall x \in \mathbb{R}^{d}, \mathbb{P}\left(X_{x}=\tilde{X}_{x}\right)=1 .
$$

Note that it follows that $X$ and $\tilde{X}$ have the same distribution since $\left(X_{x_{1}}, \ldots, X_{x_{k}}\right)=$ $\left(\tilde{X}_{x_{1}}, \ldots, \tilde{X}_{x_{k}}\right)$ a.s. for all $k \geq 1$ and $x_{1}, \ldots, x_{k} \in \mathbb{R}^{d}$. This implies a weaker notion.

Definition 16. Let $X=\left(X_{x}\right)_{x \in \mathbb{R}^{d}}$ be a random field. We say that $\tilde{X}=\left(\tilde{X}_{x}\right)_{x \in \mathbb{R}^{d}}$ is a version of $X$ if $X$ and $\tilde{X}$ have the same finite dimensional distributions.

We refer to Chapter 9 of [56] for the interested reader. 


\subsubsection{Hölder regularity}

Definition 17. Let $K=[0,1]^{d}$. Let $\gamma \in(0,1)$. A random field $X=\left(X_{x}\right)_{x \in \mathbb{R}^{d}}$ is $\gamma$ Hölder on $K$ if there exists a finite random variable $A$ such that a.s.

$$
\left|X_{x}-X_{y}\right| \leq A\|x-y\|^{\gamma}, \forall x, y \in K
$$

Note that it implies that $X$ is a.s. continuous on $K$. The following theorem gives a general criterion to ensure the existence of an Hölder modification, particularly helpful for Gaussian fields, generalizing the one dimensional case (see [39] p.53).

Theorem 4 (Kolmogorov-Chentsov 1956). If there exist $0<\beta<\delta$ and $C>0$ such that

$$
\mathbb{E}\left(\left|X_{x}-X_{y}\right|^{\delta}\right) \leq C\|x-y\|^{d+\beta}, \forall x, y \in K,
$$

then there exists $\tilde{X}$ a modification of $X \gamma$-Hölder on $K$, for all $\gamma<\beta / \delta$.

Let us note that the assumption clearly implies stochastic continuity of $X$ on $K$ in view of Markov's inequality. We give the constructing proof of this result.

Proof. Step 1. For $k \geq 1$ we introduce the dyadic points of $[0,1]^{d}$

$$
\mathscr{D}_{k}=\left\{\frac{j}{2^{k}} ; j=\left(j_{1}, \ldots, j_{d}\right) \in \mathbb{N}^{d} \text { with } 0 \leq j_{i} \leq 2^{k} \text { for all } 1 \leq i \leq d\right\} .
$$

Note that for $x \in[0,1]^{d}$, there exists $x_{k} \in \mathscr{D}_{k}$ with $\left\|x-x_{k}\right\|_{\infty} \leq 2^{-k}$ so that $\mathscr{D}_{k}$ is a $2^{-k}$ net of $K$ for $\|\cdot\|_{\infty}$, where $\|x\|_{\infty}=\max _{1 \leq i \leq d}\left|x \cdot e_{i}\right|$. The sequence $\left(\mathscr{D}_{k}\right)_{k}$ is clearly increasing.

Let $\gamma \in(0, \beta / \delta)$. For $i, j \in\left[0,2^{k}\right]^{d} \cap \mathbb{N}^{d}$ with $i \neq j$ define the measurable set

$$
E_{i, j}^{k}=\left\{\omega \in \Omega ;\left|X_{i / 2^{k}}(\omega)-X_{j / 2^{k}}(\omega)\right|>\left\|i / 2^{k}-j / 2^{k}\right\|_{\infty}^{\gamma}\right\}
$$

By assumption and Markov's inequality

$$
\mathbb{P}\left(E_{i, j}^{k}\right) \leq 2^{-k(d+\beta-\gamma \delta)}\|i-j\|_{\infty}^{d+\beta-\gamma \delta} .
$$

Set

$$
E^{k}=\underset{(i, j) \in\left[0,2^{k}\right] ; 0<\|i-j\|_{\infty} \leq 5}{\cup} E_{i, j}^{k}
$$

It follows that

$$
\begin{aligned}
\mathbb{P}\left(E^{k}\right) & \leq 5^{d+\beta-\gamma \delta} 2^{-k(d+\beta-\gamma \delta)} \#\left\{(i, j) \in\left[0,2^{k}\right] ; 0<\|i-j\|_{\infty} \leq 5\right\} \\
& \leq 5^{d+\beta-\gamma \delta} 10^{d} 2^{-k(\beta-\gamma \delta)} .
\end{aligned}
$$

Hence, by Borel-Cantelli Lemma we get $\mathbb{P}\left(\limsup _{k} E^{k}\right)=0$ so that the event $\tilde{\Omega}=$ $\cup_{k} \cap_{l \geq k} \Omega \backslash E^{l}$ satisfies $\mathbb{P}(\tilde{\Omega})=1$. Hence, for $\omega \in \tilde{\Omega}$, there exists $k^{*}(\omega)$ such that for all $l \geq k^{*}(\omega)$ and $x, y \in \mathscr{D}_{l}$ with $0<\|x-y\|_{\infty} \leq 5 \times 2^{-l}$, we have 


$$
\left|X_{x}(\omega)-X_{y}(\omega)\right| \leq\|x-y\|_{\infty}^{\gamma} .
$$

Step 2. Let us set $\mathscr{D}=\cup_{k} \mathscr{D}_{k}$. For $x, y \in \mathscr{D}$ with $0<\|x-y\|_{\infty} \leq 2^{-k^{*}(\omega)}$, there exists a unique $l \geq k^{*}(\omega)$ with

$$
2^{-(l+1)}<\|x-y\|_{\infty} \leq 2^{-l} .
$$

Moreover, one can find $n \geq l+1$ such that $x, y \in \mathscr{D}_{n}$ and for all $k \in[l, n-1]$, there exist $x_{k}, y_{k} \in \mathscr{D}_{k}$ with $\left\|x-x_{k}\right\|_{\infty} \leq 2^{-k}$ and $\left\|y-y_{k}\right\|_{\infty} \leq 2^{-k}$. We set $x_{n}=x$ and $y_{n}=y$. Therefore

$$
\begin{aligned}
\left\|x_{l}-y_{l}\right\|_{\infty} & \leq\left\|x_{l}-x\right\|_{\infty}+\|x-y\|_{\infty}+\left\|y-y_{l}\right\|_{\infty} \\
& \leq 2 \times 2^{-l}+\|x-y\|_{\infty} .
\end{aligned}
$$

But $2^{-l}<2\|x-y\|_{\infty}$ and $\left\|x_{l}-y_{l}\right\|_{\infty} \leq 5\|x-y\|_{\infty} \leq 5 \times 2^{-l}$ and since $l \geq k^{*}(\omega)$

$$
\left|X_{x_{l}}(\omega)-X_{y_{l}}(\omega)\right| \leq\left\|x_{l}-y_{l}\right\|_{\infty}^{\gamma} \leq 5^{\gamma}\|x-y\|_{\infty}^{\gamma} .
$$

But for all $k \in[l, n-1],\left\|x_{k}-x_{k+1}\right\|_{\infty} \leq 2^{-k}+2^{-(k+1)} \leq 3 \times 2^{-(k+1)}$ so that

$$
\left|X_{x_{k}}(\omega)-X_{x_{k+1}}(\omega)\right| \leq\left\|x_{k}-x_{k+1}\right\|_{\infty}^{\gamma} \leq(3 / 2)^{\gamma} 2^{-k \gamma} .
$$

Similarly,

$$
\left|X_{y_{k}}(\omega)-X_{y_{k+1}}(\omega)\right| \leq\left\|y_{k}-y_{k+1}\right\|_{\infty}^{\gamma} \leq(3 / 2)^{\gamma} 2^{-k \gamma} .
$$

It follows that

$$
\begin{aligned}
\left|X_{x}(\omega)-X_{y}(\omega)\right| & \leq \sum_{k=l}^{n-1}\left|X_{x_{k}}(\omega)-X_{x_{k+1}}(\omega)\right|+\left|X_{x_{l}}(\omega)-X_{y_{l}}(\omega)\right|+\sum_{k=l}^{n-1}\left|X_{y_{k}}(\omega)-X_{y_{k+1}}(\omega)\right| \\
& \leq \frac{2 \times 3^{\gamma}}{2^{\gamma}-1} \times 2^{-l \gamma}+5^{\gamma}\|x-y\|_{\infty}^{\gamma} \\
& \leq c_{\gamma}\|x-y\|_{\infty}^{\gamma} .
\end{aligned}
$$

Step 3. By chaining, we obtain that for all $x, y \in \mathscr{D}$

$$
\left|X_{x}(\omega)-X_{y}(\omega)\right| \leq c_{\gamma} 2^{k^{*}(\omega)}\|x-y\|_{\infty}^{\gamma},
$$

and we set $A(\omega)=c_{\gamma} 2^{k^{*}(\omega)}$. Hence we have proven that for all $\omega \in \tilde{\Omega}, x, y \in \mathscr{D}$,

$$
\left|X_{x}(\omega)-X_{y}(\omega)\right| \leq A(\omega)\|x-y\|_{\infty}^{\gamma} .
$$

We set $\tilde{X}_{x}(\omega)=0$ if $\omega \notin \tilde{\Omega}$. For $\omega \in \tilde{\Omega}$, if $x \in \mathscr{D}$ we set $\tilde{X}_{x}(\omega)=X_{x}(\omega)$. Otherwise, there exists $\left(x_{k}\right)_{k}$ a sequence of dyadic points such that $x_{k} \rightarrow x$. Therefore $\left(X_{x_{k}}(\omega)\right)$ is a Cauchy sequence and we define $\tilde{X}_{x}(\omega)$ as its limit. By stochastic continuity we have

$$
\mathbb{P}\left(\tilde{X}_{x}=X_{x}\right)=1
$$


ensuring that $\tilde{X}$ is a modification.

In order to get the best regularity we can use the notion of critical Hölder exponent, as defined in [16].

\subsubsection{Critical Hölder exponent}

Definition 18. Let $\gamma \in(0,1)$. A random field $\left(X_{x}\right)_{x \in \mathbb{R}^{d}}$ admits $\gamma$ as critical Hölder exponent on $[0,1]^{d}$ if there exists $\tilde{X}$ a modification of $X$ such that:

(a) $\forall s<\gamma$, a.s. $\tilde{X}$ satisfies $H(s): \exists A \geq 0$ a finite random variable such that $\forall x, y \in$ $[0,1]^{d}$,

$$
\left|X_{x}-X_{y}\right| \leq A\|x-y\|^{s} .
$$

(b) $\forall s>\gamma$, a.s. $\tilde{X}$ fails to satisfy $H(s)$.

For centered Gaussian random fields it is enough to consider second order regularity property as stated in the next proposition (see also [1]).

Proposition 9. Let $\left(X_{x}\right)_{x \in \mathbb{R}^{d}}$ be a centered Gaussian random field. If for all $\varepsilon>0$, there exist $c_{1}, c_{2}>0$, such that, for all $x, y \in[0,1]^{d}$,

$$
c_{1}\|x-y\|^{2 \gamma+\varepsilon} \leq \mathbb{E}\left(X_{x}-X_{y}\right)^{2} \leq c_{2}\|x-y\|^{2 \gamma-\varepsilon},
$$

then the critical Hölder exponent of $X$ on $[0,1]^{d}$ is equal to $\gamma$.

Proof. The upper bound allows to use Kolmogorov-Chentsov theorem since for all $k \in \mathbb{N}^{*}$ and $\varepsilon>0$, using the fact that $X$ is Gaussian, one can find $c$ with

$$
\mathbb{E}\left(X_{x}-X_{y}\right)^{2 k}=\frac{(2 k-1) !}{2^{k-1}(k-1) !}\left(\mathbb{E}\left(X_{x}-X_{y}\right)^{2}\right)^{k} \leq c\|x-y\|^{2 \gamma k-\varepsilon} .
$$

Hence considering $\tilde{X}$ the modification of $X$ constructed in the previous proof we see that $\tilde{X}$ is a.s. $s$-Hölder for all $s<\frac{2 \gamma k-\varepsilon}{2 k}$. But since $\frac{2 \gamma k-\varepsilon}{2 k} \rightarrow \gamma$, this is true for all $s<\gamma$. Note that according to the lower bound, for any $s>\gamma$, choosing $\varepsilon=s-\gamma$, for any $x \neq y$, the Gaussian random variable $\frac{\tilde{X}_{x}-\tilde{X}_{y}}{\|x-y\|^{s}}$ admits a variance greater than $c_{1}\|x-y\|^{-(s-\gamma)}$, that tends to infinity as $x$ tends to $y$. Therefore it is almost surely unbounded as $\|x-y\| \rightarrow 0$.

We have therefore a very simple condition on variogram for Gaussian random fields with stationary increments.

Corollary 2. Let $X$ be a centered Gaussian field with stationary increments. If for all $\varepsilon>0$, there exist $c_{1}, c_{2}>0$, such that for all $x \in[-1,1]^{d}$,

$$
c_{1}\|x\|^{2 \gamma+\varepsilon} \leq v_{X}(x)=\mathbb{E}\left(\left(X_{x}-X_{0}\right)^{2}\right) \leq c_{2}\|x\|^{2 \gamma-\varepsilon},
$$

then $X$ has critical Hölder exponent on $[0,1]^{d}$ equal to $\gamma$. 
This allows to compute critical exponents of several examples presented above.

- Fractional Brownian fields with variogram $v_{H}(x)=\|x\|^{2 H}$ and 2-dimensional elementary anisotropic fractional Brownian fields with variogram $v_{H, \alpha}(x)=$ $c_{H, \alpha}(x /\|x\|)\|x\|^{2 H}$ for $\alpha \in(0, \pi / 2]$ have critical Hölder exponent given by $H$ (see Figures 1.2.3 and 7).

- Stationary Ornstein Uhlenbeck processes have variogram given by $v_{X}(t)=$ $2\left(c_{X}(0)-c_{X}(t)\right)=2\left(1-e^{-\theta|t|}\right)$. It follows that their critical Hölder exponent is given by $1 / 2$ as for Brownian motion (see Figure 3 ).

- Operator scaling random fields with variogram given by $v_{H, E}(x)=\left(\sum_{i=1}^{d}\left|x \cdot \theta_{i}\right|^{2 \alpha_{i}}\right)^{H}$ admit $H \min _{1 \leq i \leq d} \alpha_{i}$ for critical Hölder exponent (see Figures 9 and 10).

Note that this global regularity does not capture anisotropy of these last random fields. In order to enlighten it we can consider regularity along lines.

\subsubsection{Directional Hölder regularity}

Considering a centered Gaussian random field with stationary increments $X$, one can extract line processes by restricting values along some lines. For $x_{0} \in \mathbb{R}^{d}$ and $\theta \in S^{d-1}$, the line process is defined by $L_{x_{0}, \theta}(X)=\left(X\left(x_{0}+t \theta\right)\right)_{t \in \mathbb{R}}$. It is now a onedimensional centered Gaussian process with stationary increments and variogram given by $v_{\theta}(t)=\mathbb{E}\left(\left(X\left(x_{0}+t \theta\right)-X\left(x_{0}\right)\right)^{2}\right)=v_{X}(t \theta)$.

Definition 19 ([16]). Let $\theta \in S^{d-1}$. We say that $X$ admits $\gamma(\theta) \in(0,1)$ as directional regularity in the direction $\theta$ if, for all $\varepsilon>0$, there exist $c_{1}, c_{2}>0$, such that

$$
c_{1}|t|^{2 \gamma(\theta)+\varepsilon} \leq v_{\theta}(t)=v_{X}(t \theta) \leq c_{2}|t|^{2 \gamma(\theta)-\varepsilon}, \forall t \in[-1,1] .
$$

It follows that the process $L_{x_{0}, \theta}(X)$ admits $\gamma(\theta)$ as critical Hölder exponent. Actually, by stationarity of increments, the directional regularity-if exists- may not have more than $d$ values as stated in the following proposition.

Proposition 10 ([16]). If there exists $\gamma: S^{d-1} \rightarrow(0,1)$ such that for all $\theta \in S^{d-1}$, $X$ admits $\gamma(\theta)$ as directional regularity in the direction $\theta$, then $\gamma$ takes at most $d$ values. Moreover, if $\gamma$ takes $k$ values $\gamma_{k}<\ldots<\gamma_{1}$, there exists an increasing sequence of vectorial subset $\{0\}=V_{0} \subsetneq V_{1} \subsetneq \ldots \subsetneq V_{k}:=\mathbb{R}^{d}$ such that

$$
\gamma(\theta)=\gamma_{i} \Leftrightarrow \theta \in\left(V_{i} \backslash V_{i-1}\right) \cap S^{d-1} .
$$

In our previous examples we get the following results.

- For fractional Brownian fields with variogram $v_{H}(x)=\|x\|^{2 H}$ and 2-dimensional elementary anisotropic fractional Brownian fields with variogram $v_{H}(x)=c_{H, \alpha}(x /\|x\|)\|x\|^{2 H}$ : for all $\theta \in S^{d-1}$, the directional Hölder regularity in direction $\theta$ is given by $H$ and hence is constant. 
- For operator scaling fields with variogram $v_{H, E}(x)=\left(\sum_{i=1}^{d}\left|x \cdot \theta_{i}\right|^{2 \alpha_{i}}\right)^{H}$, for all $1 \leq i \leq d$, the directional Hölder regularity in direction $\tilde{\theta}_{i}$ is given by $H \alpha_{i}$ where $\tilde{\theta}_{i}$ is an eigenvector of $E$ associated with the eigenvalue $\alpha_{i}^{-1}$ ie $E \tilde{\theta}_{i}=\alpha_{i}^{-1} \tilde{\theta}_{i}$. Moreover, assuming for instance that $\alpha_{1}>\alpha_{2}>\ldots>\alpha_{d}$, the strict subspaces defined by $V_{k}=\operatorname{span}\left(\alpha_{1}, \ldots, \alpha_{k}\right)$ for $1 \leq k \leq d$ illustrate the previous proposition by choosing $\gamma_{k}=H \alpha_{k}$ for $1 \leq k \leq d$. For example, in Figure 10, the greater directional regularity is given by $H \alpha_{2}=0.4$ only in vertical directions $\theta= \pm e_{2}$. For any other direction, the directional regularity is given by $H \alpha_{1}=0.3$. We refer to [44] for more precise results and to [24] for a general setting.

Hölder regularity properties are often linked with fractal properties as developed in the following section.

\subsection{Hausdorff dimension of graphs}

We will see in this section how to compute Hausdorff dimension of some Gaussian random fields graphs. The fractal nature comes from the fact that the dimension will not be an integer as usual. We shall recall basic facts on Hausdorff measures and dimensions before.

\subsubsection{Hausdorff measures and dimensions}

We follow [27] Chapter 2 for these definitions. Let $U \subset \mathbb{R}^{d}$ be a bounded Borel set and $\|\cdot\|$ be a norm on $\mathbb{R}^{d}$. For $\delta>0$, a finite or countable collection of subsets $\left(B_{i}\right)_{i \in I}$ of $\mathbb{R}^{d}$ is called a $\delta$-covering of $U$ if $\operatorname{diam}\left(B_{i}\right) \leq \delta$ for all $i$ and $U \subset \cup_{i \in I} B_{i}$. Then, for $s \geq 0$, we set

$$
\mathscr{H}_{\delta}^{s}(U)=\inf \left\{\sum_{i \in I} \operatorname{diam}\left(B_{i}\right)^{s} ;\left(B_{i}\right)_{i \in I} \delta \text { - covering of } U\right\} .
$$

Note that for all $\delta<\delta^{\prime}$, since a $\delta$ covering is also a $\delta^{\prime}$-covering we get $\mathscr{H}_{\delta}^{s}(U) \geq$ $\mathscr{H}_{\delta^{\prime}}^{s}(U)$. The sequence $\left(\mathscr{H}_{\delta}^{s}(U)\right)_{\delta}$ being monotonic we can give the following definition.

Definition 20. The $s$-dimensional Hausdorff measure of $U$ is defined by

$$
\mathscr{H}^{s}(U)=\lim _{\delta \rightarrow 0} \mathscr{H}_{\delta}^{s}(U) \in[0,+\infty] .
$$

Note that Hausdorff measures define measures on $\left(\mathbb{R}^{d}, \mathscr{B}\left(\mathbb{R}^{d}\right)\right)$ that generalize Lebesgue measures so that $\mathscr{H}^{1}(U)$ gives the length of a curve $U, \mathscr{H}^{2}(U)$ gives the area of a surface $U$, etc... Let us also remark that for $s^{\prime}>s$ and $\left(B_{i}\right)_{i \in I}$ a $\delta$-covering of $U$ we easily see that for all $\delta>0$ 


$$
\sum_{i \in I} \operatorname{diam}\left(B_{i}\right)^{s^{\prime}} \leq \delta^{s^{\prime}-s} \sum_{i \in I} \operatorname{diam}\left(B_{i}\right)^{s}
$$

so that $\mathscr{H}_{\delta}^{s^{\prime}}(U) \leq \delta^{s^{\prime}-s} \mathscr{H}_{\delta}^{s}(U) \leq \delta^{s^{\prime}-s} \mathscr{H}^{s}(U)$. Hence, if $\mathscr{H}^{s}(U)<+\infty$, we get $\mathscr{H}^{s^{\prime}}(U)=0$. Conversely, if $\mathscr{H}^{s^{\prime}}(U)>0$, we obtain that $\mathscr{H}^{s}(U)=+\infty$. Actually, the function $s \in[0,+\infty) \mapsto \mathscr{H}^{s}(U)$ jumps from $+\infty$ to 0 .

Definition 21. The Hausdorff dimension of $U$ is defined as

$$
\operatorname{dim}_{H}(U)=\inf \left\{s \geq 0 ; \mathscr{H}^{s}(U)=0\right\}=\sup \left\{s \geq 0 ; \mathscr{H}^{s}(U)=+\infty\right\} .
$$

Let us emphasize that in general we do not know the value of $\mathscr{H}^{s^{*}}(U) \in[0,+\infty]$ at $s^{*}=\operatorname{dim}_{H}(U)$. But we always have that $\mathscr{C}^{s}(U)>0$ implies $\operatorname{dim}_{H}(U) \geq s$, while $\mathscr{H}^{s}(U)<+\infty$ implies that $\operatorname{dim}_{H}(U) \leq s$, allowing to compute $\operatorname{dim}_{H}(U)$.

For instance, when $U=[0,1]^{d}$, choosing $\|\cdot\|_{\infty}$, we can cover $U$ by cubes $\delta i+$ $[0, \delta]^{d}$ of diameter $\delta$, for $i \in \mathbb{N}^{d}$ satisfying $0 \leq i_{k} \leq \delta^{-1}-1$. Hence we need around $\delta^{-d}$ such cubes to cover $U$ so that $\mathscr{H}_{\delta}^{s}(U) \leq c \delta^{-d} \times \delta^{s}$. It follows that for $s \geq d$, $\mathscr{H}^{s}(U)<+\infty$ and $\operatorname{dim}_{H}(U) \leq d$. But if $\left(B_{i}\right)_{i \in I}$ is a $\delta$ covering of $U$ with $\operatorname{diam}\left(B_{i}\right)=$ $r_{i}$ we get $1=\mathscr{L} e b(U) \leq \sum_{i \in I} r_{i}^{d}$ and therefore $\mathscr{H}^{d}(U)>0$ and $\operatorname{dim}_{H}(U) \geq d$. In conclusion we obtain that $\operatorname{dim}_{H}(U)=d$. This can be generalized as in the following Proposition (see [27] for instance).

Proposition 11. If $U$ is a non-empty open bounded set of $\mathbb{R}^{d}$ then $\operatorname{dim}_{H}(U)=d$.

Following similar computations we can deduce an upper bound of Hausdorff dimension for graphs of Hölder functions.

\subsubsection{Upper bound of graphs Hausdorff dimension}

Let $f:[0,1]^{d} \rightarrow \mathbb{R}$ and denote its graph by

$$
\mathscr{G}_{f}=\left\{(x, f(x)) ; x \in[0,1]^{d}\right\} \subset \mathbb{R}^{d+1} .
$$

Note that we clearly have $\operatorname{dim}_{H} \mathscr{G}_{f} \geq d$. An upper bound may be set according to the Hölder regularity of the function.

Proposition 12. If there exist $\gamma \in(0,1]$ and $C>0$ such that for all $x, y \in[0,1]^{d}$ one has $|f(x)-f(y)| \leq C\|x-y\|_{\infty}^{\gamma}$, then $\operatorname{dim}_{H} \mathscr{G}_{f} \leq d+1-\gamma$.

Proof. Write $[0,1]^{d} \subset \bigcup_{i=1}^{N_{\delta}}\left(x_{i}+[0, \delta]^{d}\right)$, where we can choose $N_{\delta}$ of the order of $\delta^{-d}$ and $x_{i} \in \delta \mathbb{N}^{d}$ for $1 \leq i \leq N_{\delta}$ as previously. Then

$$
\begin{aligned}
\mathscr{G}_{f} & \subset \bigcup_{i=1}^{N_{\delta}}\left(x_{i}+[0, \delta]^{d}\right) \times\left(f\left(x_{i}\right)+\left[-C \delta^{\gamma}, C \delta^{\gamma}\right]\right) \\
& \subset \bigcup_{i=1}^{N_{\delta}} \bigcup_{j=1}^{N_{\delta}^{\gamma}}\left(x_{i}+[0, \delta]^{d}\right) \times\left(f\left(x_{i}\right)+I_{j}(\delta)\right),
\end{aligned}
$$


choosing $N_{\delta}^{\gamma}$ intervals $\left(I_{j}(\delta)\right)_{j}$ of size $\delta$ to cover $\left[-C \delta^{\gamma}, C \delta^{\gamma}\right]$, with $N_{\delta}^{\gamma}$ of the order of $\delta^{\gamma-1}$. Hence $\mathscr{H}_{\delta}^{s}\left(\mathscr{G}_{f}\right) \leq N_{\delta} N_{\delta}^{\gamma} \delta^{s} \leq c \delta^{-d+\gamma-1+s}$. Therefore choosing $s>d+1-\gamma$ implies that $\mathscr{H}^{s}\left(\mathscr{G}_{f}\right)=0$ and $\operatorname{dim}_{H} \mathscr{G}_{f} \leq s$. Since this holds for all $s<d+1-\gamma$ we obtain that $\operatorname{dim}_{H} \mathscr{G}_{f} \leq d+1-\gamma$.

Lower bounds for Hausdorff dimension are usually more difficult to obtain.

\subsubsection{Lower bound of graphs Hausdorff dimension}

One usual way to get lower bounds is to use Frostman criteria [27]. For second order random fields, this can be formulated as in the following theorem (see Lemma 2 of [3]).

Theorem 5. Let $\left(X_{x}\right)_{x \in \mathbb{R}^{d}}$ be a second order field a.s. continuous on $[0,1]^{d}$ such that there exists $s \in(d, d+1]$,

$$
\int_{[0,1]^{d} \times[0,1]^{d}} \mathbb{E}\left(\left(\left|X_{x}-X_{y}\right|^{2}+\|x-y\|^{2}\right)^{-s / 2}\right) d x d y<+\infty,
$$

then a.s. $\operatorname{dim}_{H} \mathscr{G}_{X} \geq s$.

For Gaussian random fields this can be used in the following way.

Corollary 3. Let $(X(x))_{x \in \mathbb{R}^{d}}$ be a Gaussian random field. If there exists $\gamma \in(0,1)$ such that for all $\varepsilon>0$, there exist $c_{1}, c_{2}>0$,

$$
c_{1}\|x-y\|^{2 \gamma+\varepsilon} \leq \mathbb{E}(X(x)-X(y))^{2} \leq c_{2}\|x-y\|^{2 \gamma-\varepsilon},
$$

then, for any continuous modification $\tilde{X}$ of $X$, one has

$$
\operatorname{dim}_{H} \mathscr{G}_{\tilde{X}}=d+1-\gamma \text { a.s. }
$$

Proof. The upper bound allows to construct a modification $\tilde{X}$ of $X$ that is $\beta$ Hölder on $[0,1]^{d}$ for any $\beta<\gamma$ in view of Kolmogorov-Chentsov theorem so that $\operatorname{dim}_{H} \mathscr{G}_{\tilde{X}} \leq d+1-\gamma$ a.s. according to the previous part. Following [3] and [4], since $X$ is Gaussian, one can prove that for any $s>1$ and $\beta>\gamma$, there exists $c>0$ such that

$$
\mathbb{E}\left(\left(\left|X_{x}-X_{y}\right|^{2}+\|x-y\|^{2}\right)^{-s / 2}\right) \leq c\|x-y\|^{1-\beta-s},
$$

using the fact that $\mathbb{E}(X(x)-X(y))^{2} \geq c_{1}\|x-y\|^{2 \beta}$ by assumption. It follows that for $1-\beta-s+d>0$ the integral in Theorem 5 is finite. Hence a.s. $\operatorname{dim}_{H} \mathscr{G}_{\tilde{X}} \geq s$. Since this holds for all $\beta>\gamma$ and $s<d+1-\beta$ we get the desired lower bound and then the result.

Note that in particular we obtain that the Hausdorff dimension of fractional Brownian fields graphs, respectively 2-dimensional elementary anisotropic fractional Brownian fields graphs, with Hurst parameter $H \in(0,1)$, is given by $d+$ 
$1-H \notin \mathbb{N}$, respectively $2+1-H \notin \mathbb{N}$. For $(E, H)$-operator scaling random fields graphs, it is given by $d+1-H \min _{1 \leq i \leq d} \alpha_{i} \notin \mathbb{N}$, where $\left(\alpha_{i}^{-1}\right)_{1 \leq i \leq d}$ are the eigenvalues of $E$ (see [12]).

\section{Simulation and estimation}

This section focuses on some exact methods of simulation for some previously studied Gaussian random fields. We also give one way of estimation for fractal roughness and some applications in medical imaging.

\subsection{Simulation}

In order to simulate a centered Gaussian random field $X$ on $[0,1]^{d}$ for instance, the first step is to choose the mesh of discretization, let say $1 / n$ for some $n \in \mathbb{N}$. Then one want to simulate the centered Gaussian random vector $\left(X_{k / n}\right)_{k \in[[0, n]]^{d}}$ that is of size $(n+1)^{d}$. Choleski's method for diagonalizing the covariance matrix becomes quickly unpractical as $n$ increases. Some helpful results concerning diagonalization of circulant matrices by discrete Fourier transforms may be sometimes used under an assumption of stationarity implying a Toeplitz structure of the covariance matrix. We refer to [26] for general framework and only illustrate these ideas to set fast and exact algorithms for some fractional or operator scaling 2-dimensional fields. The first step is to simulate one-dimensional fractional Brownian motion.

\subsubsection{Fast and exact synthesis of fractional Brownian motion}

Let $H \in(0,1)$ and $B_{H}=\left(B_{H}(t)\right)_{t \in \mathbb{R}}$ be a fractional Brownian motion and recall that for $n \in \mathbb{N}$ we want to simulate $\left(B_{H}(k / n)\right)_{0 \leq k \leq n}$. By self-similarity, $\left(B_{H}(k / n)\right)_{0 \leq k \leq n} \stackrel{d}{=}$ $n^{-H}\left(B_{H}(k)\right)_{0 \leq k \leq n}$, with $B_{H}(k)=\sum_{j=0}^{k-1}\left(B_{H}(j+1)-B_{H}(j)\right)$ for $k \geq 1$, since $B_{H}(0)=0$ a.s. Hence, let us define the fractional Gaussian noise as $Y_{j}=B_{H}(j+1)-B_{H}(j)$, for $j \in \mathbb{Z}$. Since $B_{H}$ has stationary increments, $\left(Y_{j}\right)_{j \in \mathbb{Z}}$ is a centered stationary Gaussian sequence with covariance given by

$$
c_{k}=\operatorname{Cov}\left(Y_{k+j}, Y_{j}\right)=\frac{1}{2}\left(|k+1|^{2 H}-2|k|^{2 H}+|k-1|^{2 H}\right), \forall k \in \mathbb{Z} .
$$


It follows that the Gaussian vector $Y=\left(Y_{0}, \ldots, Y_{n}\right)$ has a Toeplitz covariance matrix given by $K_{Y}=\left(\begin{array}{cccc}c_{0} & c_{1} & \ldots & c_{n} \\ & \ddots & & \vdots \\ & & \ddots & c_{1} \\ & & & c_{0}\end{array}\right)$. The idea of the circulant embedding matrix method [26] is to embed $K_{Y}$ in the symmetric circulant matrix $S=\operatorname{circ}(s)$ of size $2 n$ with

$$
s=\left(c_{0} c_{1} \ldots c_{n} c_{n-1} \ldots c_{1}\right)=\left(s_{0} s_{1} \ldots s_{n} s_{n+1} \ldots s_{2 n-1}\right),
$$

and more precisely

$$
S=\left(\begin{array}{ccccc}
s_{0} & s_{2 n-1} & \ldots & s_{2} & s_{1} \\
s_{1} & s_{0} & s_{2 n-1} & & s_{2} \\
\vdots & s_{1} & s_{0} & \ddots & \vdots \\
s_{2 n-2} & & \ddots & \ddots & s_{2 n-1} \\
s_{2 n-1} & s_{2 n-2} & \ldots & s_{1} & s_{0}
\end{array}\right)=\left(\begin{array}{cc}
K_{Y} & S_{1} \\
S_{1}^{t} & S_{2}
\end{array}\right)
$$

Then $S=\frac{1}{2 n} F_{2 n}^{*} \operatorname{diag}\left(F_{2 n} S\right) F_{2 n}$ with $F_{2 n}=\left(e^{\frac{2 i \pi(j-1)(k-1)}{2 n}}\right)_{1 \leq j, k \leq 2 n}$ the matrix of discrete Fourier transform.

The symmetric matrix $S$ may be used as a covariance matrix as soon as its eigenvalues are non-negative, which is equivalent to the fact that $F_{2 n} s \geq 0$. This is in general difficult to establish and sometimes only checked numerically. However as far as fractional Gaussian noises are concerned we have a theoretical positive result established in $[23,51]$. So we may consider a square root of $S$ given by $R_{2 n}=\frac{1}{\sqrt{2 n}} F_{2 n}^{*} \operatorname{diag}\left(F_{2 n} s\right)^{1 / 2} \in \mathscr{M}_{2 n}(\mathbb{C})$. Hence, choosing two independent centered Gaussian vectors $\varepsilon^{(1)}, \varepsilon^{(2)}$ with covariance matrix $I_{2 n}$ (hence iid marginals of standard Gaussian variables), we get using the fact that $R_{2 n} R_{2 n}^{*}=S$,

$$
R_{2 n}\left[\varepsilon^{(1)}+i \varepsilon^{(2)}\right]=Z^{(1)}+i Z^{(2)},
$$

with $Z^{(1)}, Z^{(2)}$ iid $\mathscr{N}(0, S)$. It follows that

$$
Y \stackrel{d}{=}\left(Z_{k}^{(1)}\right)_{0 \leq k \leq n} \stackrel{d}{=}\left(Z_{k}^{(2)}\right)_{0 \leq k \leq n} \sim \mathscr{N}\left(0, K_{Y}\right) .
$$

Choosing $n=2^{p}$ we can use fast discrete Fourier transforms to get a cost of simulation $O(n \log (n))$ to compare with $O\left(n^{3}\right)$ for Choleski method. On Figure 11, we can now illustrate previous results on the regularity and graphs Hausdorff dimension of fractional Brownian motions given respectively by $H$ and $2-H$ in dimension 1 .

When considering 2-dimensional Gaussian fields, several extensions are possible. For instance, if $\left(Y_{k_{1}, k_{2}}\right)_{\left(k_{1}, k_{2}\right) \in \mathbb{Z}^{2}}$ is stationary, its covariance function may be written as $\operatorname{Cov}\left(Y_{k_{1}+l_{1}, k_{2}+l_{2}}, Y_{l_{1}, l_{2}}\right)=r_{k_{1}, k_{2}}$. Hence we may use a block Toeplitz covariance matrix with Toeplitz blocks and embed in a block circulant matrix (see 


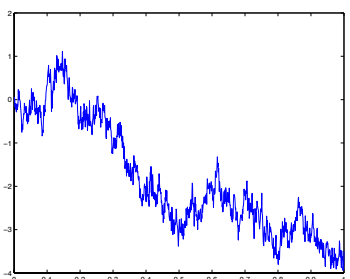

$H=0.3$

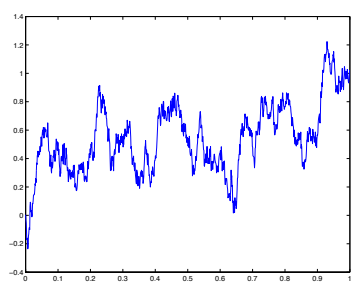

$H=0.5$

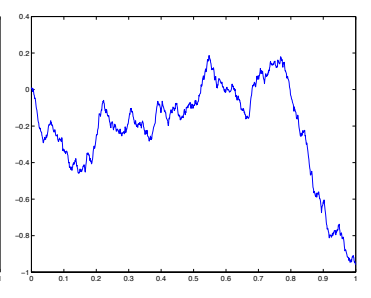

$H=0.7$

Fig. 11 Simulation of $\left(B_{H}(k / n)\right)_{0 \leq k \leq n}$ for $n=2^{12}$ using circulant embedding matrix method

$[21,26])$. When only stationary increments are assumed one can still try to simulate the increments but in dimension $d>1$ the initial conditions now correspond to values on axis and are correlated with increments [38].

We present two other possible generalizations for considered random fields based on more general ideas.

\subsubsection{Turning band method for 2-dimensional anisotropic self-similar fields}

The turning band method was introduced by Matheron in [48]. It is mainly based on similar ideas developed in Proposition 3 when considering isotropic fields constructed from processes. Actually, when $Y$ is a centered second order stationary process with covariance $K_{Y}(t, s)=c_{Y}(t-s)$ one can define the field

$$
Z(x)=Y(x \cdot U) \text { for } x \in \mathbb{R}^{2},
$$

by choosing $U \sim \mathscr{U}\left(S^{1}\right)$ independent from $Y$. It follows that $Z$ is a centered stationary isotropic field such that identifying $\theta \in[-\pi, \pi]$ with $u(\theta)=(\cos (\theta), \sin (\theta)) \in$ $S^{1}$,

$$
c_{Z}(x)=\operatorname{Cov}(Z(x+y), Z(y))=\frac{1}{\pi} \int_{-\pi / 2}^{\pi / 2} c_{Y}(x \cdot u(\theta)) d \theta .
$$

Let us note that even if $Y$ is Gaussian $Z$ is not a Gaussian field.

Assuming that we are able to simulate $Y$ one can define for $K \geq 1, \theta_{1}, \ldots, \theta_{K} \in$ $[-\pi / 2, \pi / 2]$ and $\lambda_{1}, \ldots, \lambda_{K} \in \mathbb{R}^{+}$, an approximated field

$$
Z_{K}(x)=\sum_{i=1}^{K} \sqrt{\lambda_{i}} Y^{(i)}\left(x \cdot u\left(\theta_{i}\right)\right)
$$

with $Y^{(1)}, \ldots, Y^{(K)}$ independent realizations of $Y$. The field $Z_{K}$ is a centered stationary field with covariance

$$
c_{Z_{K}}(x)=\sum_{i=1}^{K} \lambda_{i} c_{Y}\left(x \cdot u\left(\theta_{i}\right)\right)
$$


such that choosing convenient weights it can be an approximation of the covariance $c_{Z}$. For Gaussian random field, Matheron proposes to use the central limit theorem and considers $\frac{1}{\sqrt{N}}\left(Z_{K}^{(1)}+\ldots+Z_{K}^{(N)}\right)$, with $Z_{K}^{(1)}, \ldots, Z_{K}^{(N)}$ independent realizations of $Z_{K}$. In [13] we have exploited these ideas to propose simulations of anisotropic selfsimilar fields. Let $H \in(0,1), \mu$ be a finite non-negative measure on $S^{1}$, and $X_{H, \mu}=$ $\left(X_{H, \mu}(x)\right)_{x \in \mathbb{R}^{2}}$ be a centered Gaussian random field with stationary increments and variogram given by

$$
v_{H, \mu}(x)=\int_{S^{1}}|x \cdot \theta|^{2 H} \mu(d \theta)=C_{H, \mu}\left(\frac{x}{\|x\|}\right)\|x\|^{2 H} .
$$

We recall that $X_{H, \mu}$ is self-similar of order $H$. Note also that choosing $\mu$ the uniform measure on $S^{1}$, the corresponding field $X_{H, \mu}$ is isotropic and therefore it is a fractional Brownian field. When $\mu_{K}$ is a discrete measure ie $\mu_{K}=\sum_{i=1}^{K} \lambda_{i} \delta_{\theta_{i}}$ for some $\theta_{1}, \ldots, \theta_{K} \in S^{1}$ and $\lambda_{1}, \ldots, \lambda_{K} \in \mathbb{R}^{+}$, we get

$$
v_{H, \mu_{K}}(x)=\sum_{i=1}^{K} \lambda_{i}\left|x \cdot \theta_{i}\right|^{2 H}=\sum_{i=1}^{K} \lambda_{i} \operatorname{Var}\left(B_{H}\left(x \cdot \theta_{i}\right)\right) .
$$

Hence, considering, $\left(B_{H}^{(i)}\right)_{1 \leq i \leq K}$ independent realizations of the one dimensional $H$ fractional Brownian motion,

$$
X_{H, \mu_{K}}(x):=\sum_{i=1}^{K} \sqrt{\lambda_{i}} B_{H}^{(i)}\left(x \cdot \theta_{i}\right), x \in \mathbb{R}^{2},
$$

is a centered Gaussian random field with stationary increments and variogram $v_{H, \mu_{K}}$.

Hence the simulation of these random fields depends on the simulation of $B_{H}$ on some specific points. We can exploit further on this fact using specific choices of lines and weights. Actually, to simulate $\left(X_{H, \mu_{K}}\left(\frac{k_{1}}{n}, \frac{k_{2}}{n}\right)\right)_{0 \leq k_{1}, k_{2} \leq n}$ one has to simulate for $1 \leq i \leq K$,

$$
B_{H}^{(i)}\left(\frac{k_{1}}{n} \cos \left(\theta_{i}\right)+\frac{k_{2}}{n} \sin \left(\theta_{i}\right)\right) \text { for } 0 \leq k_{1}, k_{2} \leq n .
$$

When $\cos \left(\theta_{i}\right) \neq 0$, by choosing $\theta_{i}$ with $\tan \left(\theta_{i}\right)=\frac{p_{i}}{q_{i}}$ for $p_{i} \in \mathbb{Z}$ and $q_{i} \in \mathbb{N}$, using self-similarity we get

$$
\left(B_{H}^{(i)}\left(\frac{k_{1}}{n} \cos \left(\theta_{i}\right)+\frac{k_{2}}{n} \sin \left(\theta_{i}\right)\right)\right)_{k_{1}, k_{2}} \stackrel{f d d}{=}\left(\frac{\cos \left(\theta_{i}\right)}{n q_{i}}\right)^{H}\left(B_{H}^{(i)}\left(k_{1} q_{i}+k_{2} p_{i}\right)\right)_{k_{1}, k_{2}} .
$$

Using the previous algorithm we are able to simulate this with a cost given by $O\left(n\left(\left|p_{i}\right|+q_{i}\right) \log \left(n\left(\left|p_{i}\right|+q_{i}\right)\right)\right)$. For $\mu(d \theta)=c(\theta) d \theta$, in particular for elementary anisotropic fractional fields (see Figure 7), Riemann approximation for convenient 
$\mu_{K}$ yields to error bounds between the distribution of $X_{H, \mu_{K}}$ and the distribution of $X_{H, \mu}$ so that $X_{H, \mu_{K}}$ may be used as an approximation to simulate $X_{H, \mu}$. Note that contrarily to the original Turning band method, the simulated fields are all Gaussian, with stationary increments, and self-similar of order $H$. We refer to Figure 12 where we can see the effect of the number of chosen lines on induced realizations as well as on corresponding variograms.
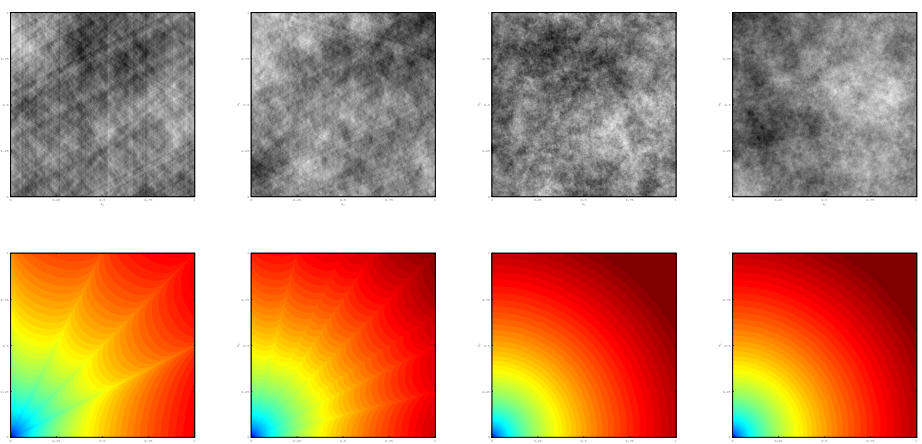

$$
K=7
$$

$K=15$

$K=51$

$K=103$

Fig. 12 Top: realizations of $X_{H, \mu_{K}}$ with $H=0.2$ to approximate the isotropic field $X_{H, \mu}$ for $\mu(d \theta)=d \theta$ and $n=512$; Bottom: corresponding variograms $v_{H, \mu_{K}}$ (see [13]).

Actually, a fast and exact method of simulation has been set up in [57] for isotropic fractional Brownian fields $X_{H, \mu}$. This is based on a local stationary approximation of this field by considering a stationary field with compactly supported covariance function for which the 2-dimensional circulant embedding matrix algorithm is running. These ideas have also been exploited in [29] and may be partially used for more general operator scaling random field [11]. We briefly present this in the following section.

\subsubsection{Stein method for 2-dimensional Operator Scaling fields}

For the sake of simplicity we consider here the diagonal case and set $E=\operatorname{diag}\left(\alpha_{1}^{-1}, \alpha_{2}^{-1}\right)$ for some $\alpha_{1}, \alpha_{2} \in(0,1]$ and $\tau_{E}(x)^{2}:=\left|x_{1}\right|^{2 \alpha_{1}}+\left|x_{2}\right|^{2 \alpha_{2}}$. Recall that, by Proposition 8 , for $H \in(0,1]$ we can define $X_{H, E}=\left(X_{H, E}(x)\right)_{x \in \mathbb{R}^{2}}$ a centered Gaussian random field with stationary increments and variogram given by

$$
v_{H, E}(x)=\tau_{E}(x)^{2 H}=\left(\left|x_{1}\right|^{2 \alpha_{1}}+\left|x_{2}\right|^{2 \alpha_{2}}\right)^{H},
$$

so that $X_{H, E}$ is $(E, H)$-operator scaling. Let us also note that for $\alpha_{1}=\alpha_{2}=\alpha \in(0,1]$, the field $X_{H, E}$ is $\alpha H$-self-similar but it is isotropic only when the common value $\alpha$ is equal to 1 . In this case $X_{H, E}$ corresponds to a fractional Brownian field of 
order $\alpha H=H$. In [11], we extend the procedure developed in [57] for fast and exact synthesis of these last fields. Let us define for $c_{H}=1-H$, the real compactly supported function

$$
K_{H, E}(x)=\left\{\begin{array}{cl}
c_{H}-\tau_{E}(x)^{2 H}+\left(1-c_{H}\right) \tau_{E}(x)^{2} & \text { if } \tau_{E}(x) \leq 1 \\
0 & \text { else }
\end{array} \text { for } x \in \mathbb{R}^{2} .\right.
$$

Assuming that $K_{H, E}$ is a covariance function on $\mathbb{R}^{2}$ we can define $Y_{H, E}$ a stationary centered Gaussian random field with covariance $K_{H, E}$. Then, computing covariance functions we get that

$$
\begin{gathered}
\left\{X_{H, E}(x) ; x \in[0, M]^{2}\right\} \\
\stackrel{f d d}{=}\left\{Y_{H, E}(x)-Y_{H, E}(0)+\sqrt{1-c_{H}} B_{\alpha_{1}}^{(1)}\left(x_{1}\right)+\sqrt{1-c_{H}} B_{\alpha_{2}}^{(2)}\left(x_{2}\right) ; x \in[0, M]^{2}\right\},
\end{gathered}
$$

for $M=\max \left\{0 \leq r \leq 1 ; r^{2 \alpha_{1}}+r^{2 \alpha_{2}} \leq 1\right\}$ and $B_{\alpha_{1}}^{(1)}, B_{\alpha_{2}}^{(2)}$ two standard independent 1-dimensional fractional Brownian motions, independent from $Y_{H, E}$. Since we are already able to simulate $\left(B_{\alpha_{i}}^{(i)}(k / n)\right)_{0 \leq k \leq n}$ in view of Section 3.1.1, it is enough to simulate $\left(Y_{H, E}\left(k_{1} / n, k_{2} / n\right)\right)_{0 \leq k_{1}, k_{2} \leq n}$ in order to simulate $\left(X_{H, E}\left(k_{1} / n, k_{2} / n\right)\right)_{0 \leq k_{1}, k_{2} \leq M n}$ But if $K_{H, E}$ is a covariance function, since it has compact support in $[-1,1]^{2}$, its periodization

$$
K_{H, E}^{p e r}(x)=\sum_{k_{1}, k_{2} \in \mathbb{Z}^{2}} K_{H, E}\left(x_{1}+2 k_{1}, x_{2}+2 k_{2}\right),
$$

will also be a periodic covariance function on $\mathbb{R}^{2}$. Denoting by $Y_{H, E}^{\text {per }}$ a stationary periodic centered Gaussian random field with covariance function $K_{H, E}^{p e r}$ and remarking that $K_{H, E}(x)=K_{H, E}\left(\left|x_{1}\right|,\left|x_{2}\right|\right)$, the random vector $\left(Y_{H, E}^{p e r}\left(\frac{k_{1}}{n}, \frac{k_{2}}{n}\right)\right)_{0 \leq k_{1}, k_{2}<2 n}$ has a block circulant covariance matrix diagonalized by $2 \mathrm{D}$ discrete Fourier transform. Following [26], a fast and exact synthesis of $\left(Y_{H, E}\left(\frac{k_{1}}{n}, \frac{k_{2}}{n}\right)\right)_{0 \leq k_{1}, k_{2} \leq n} \stackrel{d}{=}$ $\left(Y_{H, E}^{\text {per }}\left(\frac{k_{1}}{n}, \frac{k_{2}}{n}\right)\right)_{0 \leq k_{1}, k_{2} \leq n}$ is possible with a cost of the order $O\left(n^{2} \log (n)\right)$. Note that according to Theorem 2, by the Fourier inverse theorem, the function $K_{H, E}$ is a covariance matrix if and only if its Fourier transform, defined for $\xi \in \mathbb{R}^{2}$ by $\hat{K}_{H, E}(\xi)=\int_{\mathbb{R}^{2}} e^{-i x \cdot \xi} K_{H, E}(x) d x$, is non-negative. This was proven in [57] in the isotropic case for $\alpha_{1}=\alpha_{2}=\alpha=1$ and $H \in(0,3 / 4)$. Note also that in this case we simply have $\left(B_{\alpha_{i}}^{(i)}\left(x_{i}\right)\right) \stackrel{d}{=}\left(x_{i} N^{(i)}\right)$ with $N^{(i)} \sim \mathscr{N}(0,1)$ for $i=1,2$. As long as we only want to synthesize the vector $\left(X_{H, E}\left(k_{1} / n, k_{2} / n\right)\right)_{0 \leq k_{1}, k_{2} \leq M n}$ it is actually sufficient to check numerically the non-negativeness of eigenvalues for the covariance matrix of $\left(Y_{H, E}^{p e r}\left(\frac{k_{1}}{n}, \frac{k_{2}}{n}\right)\right)_{0 \leq k_{1}, k_{2} \leq 2 n}$. We refer to Figures 13 and 14 for some realizations. 

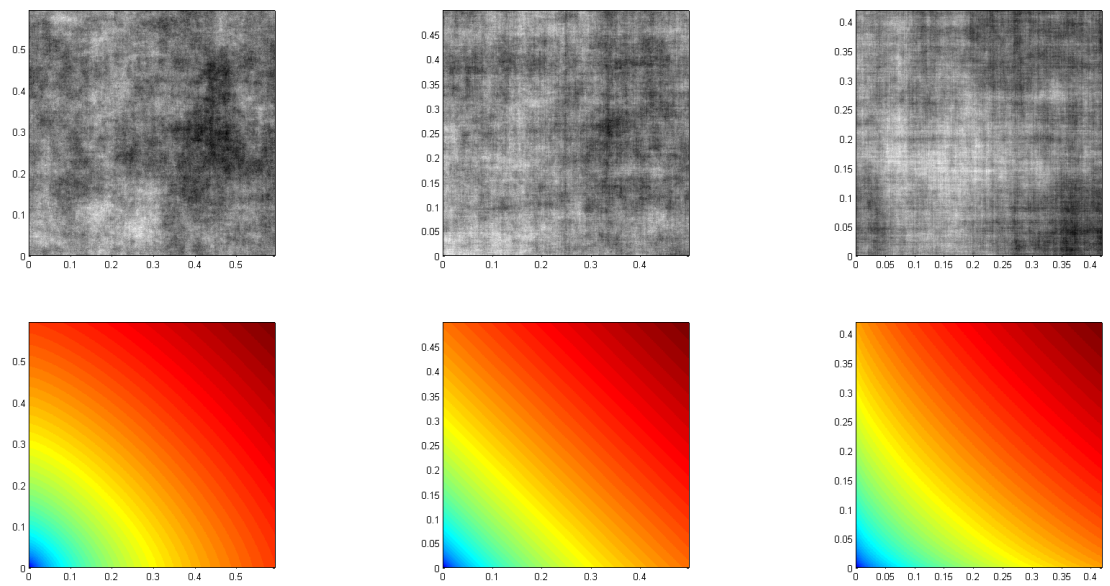

$H=0.3$

$H=0.4$

$H=0.5$

Fig. 13 Top: realizations of anisotropic self-similar fields $X_{H, E}$ with $E=\alpha^{-1} I_{2}$ and $H \alpha=0.2$ for $n=2^{10}$; Bottom: corresponding variograms $v_{H, E}$ (see [11]).
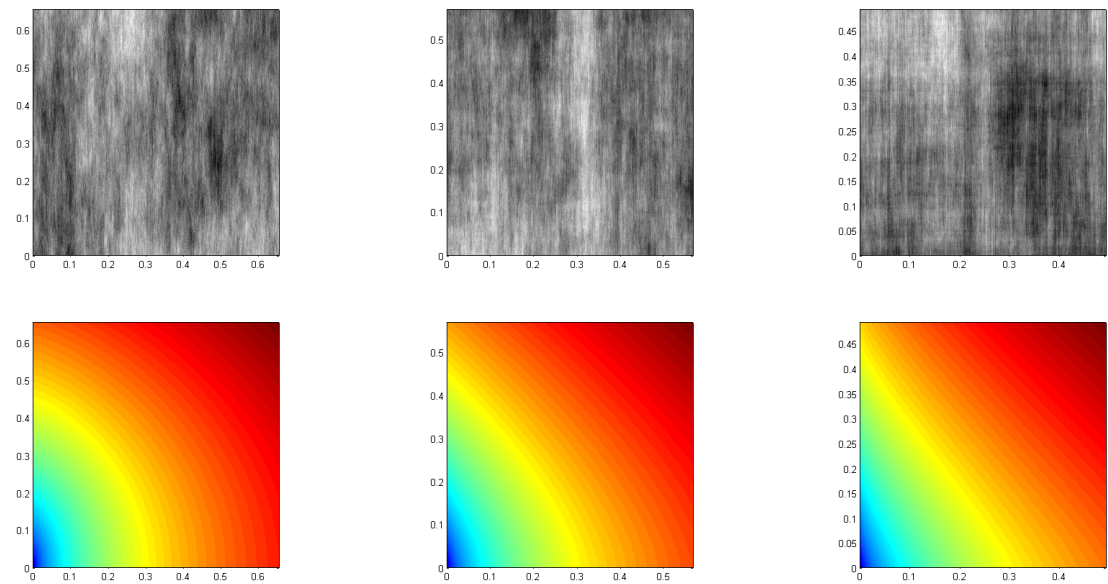

$$
H=0.3
$$

$$
H=0.4
$$

$$
H=0.5
$$

Fig. 14 Top: realizations of operator scaling fields $X_{H, E}$ with $E=\operatorname{diag}\left(\alpha_{1}^{-1}, \alpha_{2}^{-1}\right)$ and $H \alpha_{1}=0.2$, $H \alpha_{2}=0.3$ for $n=2^{10}$; Bottom: corresponding variograms $v_{H, E}$ (see [11]). 


\subsection{Estimation}

We describe here one way of estimation for Hölder directional regularity from a discretized sample paths observation. Our procedure is based on the variogram method for estimation of Hurst parameter of one-dimensional fractional Brownian motions.

\subsubsection{D estimation based on variograms}

We first assume to observe $\left(B_{H}(k)\right)_{0 \leq k \leq n}$ for some large $n$, where $B_{H}=\left(B_{H}(t)\right)_{t \in \mathbb{R}}$ is a fractional Brownian motion with Hurst parameter $H \in(0,1)$. We exploit the stationarity of increments by considering the increments of $B_{H}$ with step $u \in \mathbb{N}$

$$
\Delta_{u} B_{H}(k)=B_{H}(k+u)-B_{H}(k)
$$

Note that the sequence $\left(\Delta_{u} B_{H}(k)\right)_{k \in \mathbb{Z}}$ is a stationary Gaussian centered sequence, with common variance given by $v_{H}(u)=c_{H}|u|^{2 H}$. Hence, considering the statistics

$$
V_{n}(u)=\frac{1}{n-u} \sum_{k=0}^{n-1-u} \Delta_{u} B_{H}(k)^{2}
$$

we immediately see that $\mathbb{E}\left(V_{n}(u)\right)=v_{H}(u)$. Actually, since $\Delta_{u} B_{H}$ is Gaussian and $\operatorname{Cov}\left(\Delta_{u} B_{H}(k), \Delta_{u} B_{H}(0)\right) \underset{k \rightarrow+\infty}{\longrightarrow} 0$, this sequence is ergodic (see [45]) so that $V_{n}(u)$ is a strongly consistent estimator of $v_{H}(u)$ meaning that $V_{n}(u) \underset{n \rightarrow+\infty}{\longrightarrow} v_{H}(u)$ a.s. This naturally leads us to consider for two different steps $u \neq v$, the statistic

$$
\widehat{H}_{n}=\frac{1}{2} \log \left(\frac{V_{n}(u)}{V_{n}(v)}\right) / \log \left(\frac{u}{v}\right) .
$$

Theorem 6. For $H \in(0,1)$, the statistic $\widehat{H}_{n}$ is a strongly consistent estimator of $H$. Moreover for $H \in(0,3 / 4)$ it is also asymptotically normal.

Proof. The consistency is immediate once remarked that $H=\frac{1}{2} \log \left(\frac{v_{H}(u)}{v_{H}(v)}\right) / \log \left(\frac{u}{v}\right)$. To prove asymptotic normality, the first step is a central limit theorem for

$$
\frac{V_{n}(u)}{v_{H}(u)}=\frac{1}{n-u} \sum_{k=0}^{n-1-u} X_{u}(k)^{2} \text { with } X_{u}(k)=\frac{\Delta_{u} B_{H}(k)}{\sqrt{v_{H}(u)}},
$$

so that $\left(X_{u}(k)\right)_{k}$ is a centered stationary Gaussian sequence with unit variance. Then, denoting $H_{2}(x)=x^{2}-1$ the Hermite polynomial of order 2 we consider

$$
Q_{n}(u):=\sqrt{n-u}\left(\frac{V_{n}(u)}{v_{H}(u)}-1\right)=\frac{1}{\sqrt{n-u}} \sum_{k=0}^{n-1-u} H_{2}\left(X_{u}(k)\right)
$$


We will use a general result of Breuer Major [17] giving a summability condition on the covariance sequence to get asymptotic normality.

Proposition 13 ([17]). If $\left(\rho_{u}(k)\right)_{k}=\left(\operatorname{Cov}\left(X_{u}(k), X_{u}(0)\right)\right)_{k}$ satisfies

$$
\sigma_{u}^{2}=\sum_{k \in \mathbb{Z}} \rho_{u}(k)^{2}<+\infty, \text { then }
$$

i) $\operatorname{Var}\left(Q_{n}(u)\right) \rightarrow 2 \sigma_{u}^{2}$;

ii) $\frac{Q_{n}(u)}{\sqrt{\operatorname{Var}\left(Q_{n}(u)\right)}} \stackrel{d}{\rightarrow} N$, with $N \sim \mathscr{N}(0,1)$.

But since $X_{u}(k)=\frac{\Delta_{u} B_{H}(k)}{\sqrt{v_{H}(u)}}$ we obtain that

$$
\rho_{u}(k)=\frac{1}{2}\left(|k+u|^{2 H}-2|k|^{2 H}+|k-u|^{2 H}\right)=O_{|k| \rightarrow+\infty}\left(|k|^{-2(1-H)}\right) .
$$

Hence, we check that $\sum_{k \in \mathbb{Z}} \rho_{u}(k)^{2}<+\infty$ for $H<3 / 4$, and by Slutsky's theorem $Q_{n}(u) \stackrel{d}{\rightarrow} \mathscr{N}\left(0, \sigma_{u}^{2}\right)$. By Delta-method (see [58] for instance), asymptotic normality of $\widehat{H}_{n}$ will follow from asymptotic normality of the couple $\left(Q_{n}(u), Q_{n}(v)\right)$. Note that we already have checked it for each marginal. However since $Q_{n}(u)$ and $Q_{n}(v)$ are in the same Wiener chaos of order two we can use a very strong result of [50] saying that if $\operatorname{Cov}\left(Q_{n}(u), Q_{n}(v)\right) \rightarrow \sigma_{u v}$, asymptotic normality of marginals imply asymptotic normality of the couple namely

$$
\left(Q_{n}(u), Q_{n}(v)\right) \stackrel{d}{\rightarrow} \mathscr{N}\left(0,\left(\begin{array}{cc}
\sigma_{u}^{2} & \sigma_{u v} \\
\sigma_{u v} & \sigma_{v}^{2}
\end{array}\right)\right),
$$

that concludes the proof.

In order to get rid of the upper bound $H<3 / 4$ for asymptotic normality, Istas and Lang [36] have proposed to consider generalized quadratic variations. In particular we can replace $\Delta_{u} B_{H}(k)$ by second order increments

$$
\Delta_{u}^{(2)} B_{H}(k)=B_{H}(k+2 u)-2 B_{H}(k+u)+B_{H}(k)
$$

so that we keep a centered Gaussian stationary sequence with a similar variance given by $v_{H}^{(2)}(u)=\operatorname{Var}\left(\Delta_{u}^{(2)} B_{H}(k)\right)=c_{H}^{(2)}|u|^{2 H}$ but now the covariance sequence is $O_{k \rightarrow+\infty}\left(|k|^{-2(2-H)}\right)$ ensuring asymptotic normality for $Q_{n}^{(2)}(u)$, obtained by replacing $X_{u}$ by $X_{u}^{(2)}=\frac{\Delta_{u}^{(2)} B_{H}}{\sqrt{v_{H}^{(2)}(u)}}$ in (7) and, as a consequence for $\widehat{H}_{n}^{(2)}$ for all $H \in(0,1)$. This way of estimation is particularly robust and consistency as well as asymptotic normality still hold for infill or high frequency estimation where we now assume to observe $\left(B_{H}(k / n)\right)_{0 \leq k \leq n}$ instead of $\left(B_{H}(k)\right)_{0 \leq k \leq n}$. In this framework we have to replace $\Delta_{u}^{(2)} B_{H}(k)$ by

$$
\Delta_{u / n}^{(2)} B_{H}(k / n)=B_{H}\left(\frac{k+2 u}{n}\right)-2 B_{H}\left(\frac{k+u}{n}\right)+B_{H}\left(\frac{k}{n}\right) .
$$


Let us note that by self-similarity $\left(\Delta_{u / n}^{(2)} B_{H}(k / n)\right)_{k} \stackrel{d}{=}\left(n^{-H} \Delta_{u}^{(2)} B_{H}(k)\right)_{n}$ but we can also replace the self-similar process $B_{H}$ by $Y$ a centered Gaussian process with stationary increments such that

$$
v_{Y}(u)=\mathbb{E}\left((Y(t+u)-Y(t))^{2}\right)=c_{Y}|u|^{2 H}+O_{|u| \rightarrow 0}\left(|u|^{2 H+\varepsilon}\right) .
$$

Asymptotic normality still hold assuming that $\varepsilon>1 / 2$ but now, under our assumption, we get an estimator of Hölder regularity of $Y$, given by $H$ in view of Proposition 9. We refer to [8] for more details and to [6] for complements.

\subsubsection{Application to random fields by line processes}

This framework may be used when considering random fields $(X(x))_{x \in \mathbb{R}^{d}}$ instead of one-dimensional processes. Actually, for $x_{0} \in \mathbb{R}^{d}$ and $\theta \in S^{d-1}$ we can consider the line process $L_{x_{0}, \theta}(X)=\left\{X\left(x_{0}+t \theta\right) ; t \in \mathbb{R}\right\}$ already defined in Section 2.1.3.

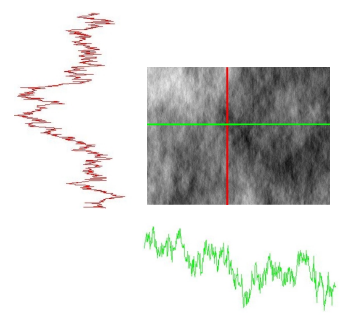

Fig. 15 A realization of a 2-dimensional random field and two line processes. In green for the horizontal direction $\theta=(1,0)$. In red for the vertical one $\theta=(0,1)$.

Recall that when $X$ is a centered stationary Gaussian random field with stationary increments and variogram $v_{X}$, the process $L_{x_{0}, \theta}(X)$ is centered Gaussian with stationary increments and variogram

$$
v_{\theta}(t)=\mathbb{E}\left(\left(X\left(x_{0}+t \theta\right)-X\left(x_{0}\right)\right)^{2}\right)=v_{X}(t \theta), t \in \mathbb{R} .
$$

When moreover $X$ is self-similar of order $H \in(0,1)$, we clearly get that $v_{\theta}(t)=$ $v_{X}(\theta)|t|^{2 H}$, ensuring that $L_{x_{0}, \theta}(X)-L_{x_{0}, \theta}(X)(0)$ is also self-similar of order $H \in$ $(0,1)$ and therefore it is a (non-standard) fractional Brownian motion with Hurst parameter $H$. Hence estimators set up in previous section may be used to estimate $H$. Note also that when $X$ is isotropic we must have $\theta \in S^{d-1} \mapsto v_{X}(\theta)$ a constant function. Finally, let us remark that considering $(E, H)$-operator scaling fields with $\alpha_{1}, \ldots, \alpha_{d} \in(0,1], H \in(0,1)$ and $E$ the diagonal matrix $\operatorname{diag}\left(\alpha_{1}^{-1}, \ldots, \alpha_{d}^{-1}\right)$, for any $1 \leq i \leq d$, the line process $L_{x_{0}, e_{i}}(X)-L_{x_{0}, e_{i}}(X)(0)$ is a fractional Brownian motion with Hurst parameter $H \alpha_{i}$, where $\left(e_{i}\right)_{1 \leq i \leq d}$ is the canonical basis of $\mathbb{R}^{d}$. This follows 
from the fact that $v_{e_{i}}(t)=v_{H, E}\left(t e_{i}\right)=|t|^{\alpha_{i} H}$ since $v_{H, E}(x)=\left(\left|x_{1}\right|^{\alpha_{1}}+\ldots+\left|x_{d}\right|^{\alpha_{d}}\right)^{2 H}$. Hence we are able to estimate $\alpha_{1} H, \ldots, \alpha_{d} H$ (see [11] for numerical results).

We can illustrate this with some applications in medical imaging.

\subsection{Application in medical imaging analysis}

There are numerous methods and studies around what is called fractal analysis in medical imaging. We refer to [46] for a good review. The main goal is to characterized self-similarity of images with a fractal index $H \in(0,1)$ to extract some helpful information for diagnosis. Our point of view consists in considering an image $\left(I\left(k_{1}, k_{2}\right)\right)_{1 \leq k_{1}, k_{2} \leq n}$ as a realization of a random field. Then,

- Extract a line from the image $\left(L_{\theta}(k)\right)_{1 \leq k \leq n_{\theta}}$ for $\theta$ a direction.

- Compute $v_{\theta}(u)=\frac{1}{n_{\theta}-u} \sum_{k=1}^{n_{\theta}-u}\left(L_{\theta}(k+u)-L_{\theta}(k)\right)^{2}$.

- Average along several lines of the same direction $\overline{v_{\theta}}(u)$ and compute $\widehat{H}_{\theta}(u, v)=$ $\frac{1}{2} \log \left(\frac{\overline{v_{\theta}}(u)}{\overline{v_{\theta}}(v)}\right) / \log \left(\frac{u}{v}\right)$.

Of course there are several implementation issues according to the chosen direction. It follows that considering estimation on oriented lines without interpolation does

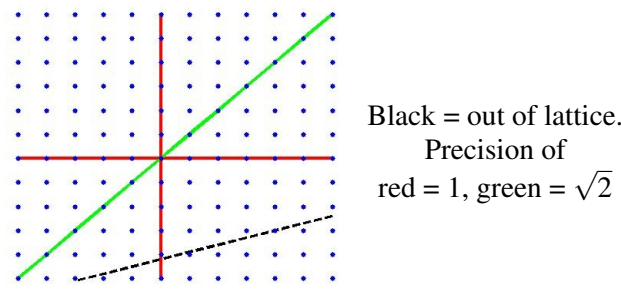

not allow to reach any direction. Moreover precision is not the same in all directions. However the procedure is successful when considering horizontal and vertical direction and may also be compared with diagonal directions. We present in the following some results obtained with two kind of medical images: bone radiographs and mammograms.

\subsubsection{Osteoporosis and bone radiographs}

According to experts from the World Health Organization, osteoporosis is a disease affecting many millions of people around the world. It is characterized by low bone mass and micro-architectural deterioration of bone tissue, leading to bone 
fragility and a consequent increase in risk of fracture. Bone mineral density allows to measure low bone mass and is used for diagnosis while micro-architectural deterioration is not quantify. Several medical research teams have been working on micro-architectural deterioration assessment from bone radiographs, an easiest and cheapest clinical exam. Several authors have proposed to use fractal analysis with different methods of analysis for instance for calcaneous bone in [31], and cancellous bone in [20]. In [5], it allows to discriminate between osteoporotic cases $H_{\text {mean }}=0.679 \pm 0.053$ and control cases $H_{\text {mean }}=0.696 \pm 0.030$, by coupling with bone mineral density.

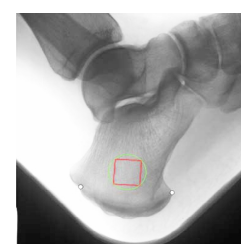

ROI

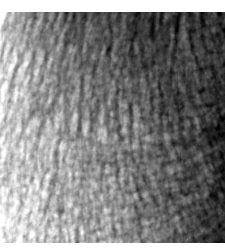

control case

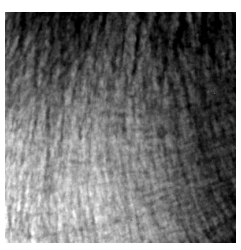

osteoporotic case

Fig. 16 Standardized acquisition of region of interest (ROI) of Inserm unit U658 [43].

In [7], we have considered a data set composed of 211 numeric radiographs highresolution of calcaneum (bone heel) with standardized acquisition of region of interest (ROI) $400 \times 400$ of Inserm unit U658 [43] (see Figure 16). Figure 17 gives the results we obtained for horizontal and vertical directions. Log-log plots are linear for small scales in adequation with a self-similarity property valid for small scales. Estimated values are greater in the vertical direction than in the horizontal direction contradicting an isotropic or self-similar model. Similar results were recently obtained using different kinds of estimators in [32]. Moreover, comparisons with diagonal direction lead us to think that operator scaling random fields could be used for modeling.

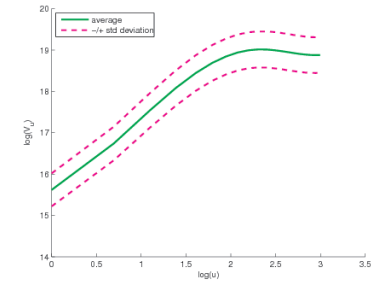

$$
\theta_{1}=(1,0), H_{\theta_{1}}=0.51 \pm 0.08
$$

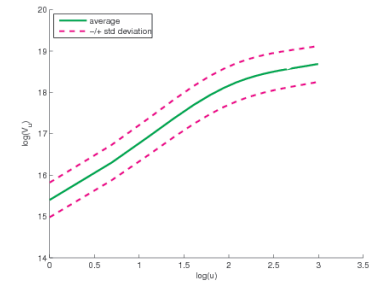

$$
\theta_{2}=(0,1), H_{\theta_{2}}=0.56 \pm 0.06
$$

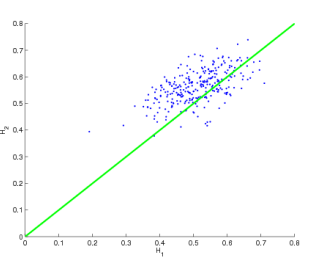

$$
H_{\theta_{2}} \text { vs } H_{\theta_{1}}
$$

Fig. 17 Mean of log-log plot of mean quadratic variations in horizontal and vertical direction. The last plot indicates estimated values of couple $\left(H_{\theta_{1}}, H_{\theta_{2}}\right)$ for each 211 images (see [7]). 


\subsubsection{Mammograms and density analysis}

Fractal analysis has also been used in mammograms analysis. In particular, it was used for the characterization and classification of mammogram density [19]. Actually, breast tissues are mainly composed of two kinds of tissues called dense and fatty (see Figure 18) and the amount of dense tissues is believed to be a risk factor for developing breast cancer $[19,33]$.
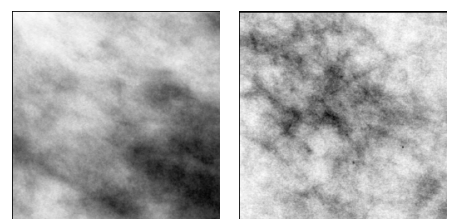

dense breast tissue fatty breast tissue

Fig. 18 Region of interest extracted from real mammograms.

In [33], the hypothesis of a self-similar behavior is validated using a power spectrum method with an estimated fractal index range $H \in[0.33,0.42]$. Based on the variogram method presented above we also found a local self-similar behavior with similar values $H=0.31 \pm 0.05$ on a data set of 58 cases with 2 mammograms (left and right) ROI of size $512 \times 512$ in [14]. Note that, contrarily to bones data, we do not have a standardized procedure to extract ROI. Very interesting results were obtained in [40] who manages to discriminate between dense and fatty breast tissues using the Wavelet Transform Modulus Maxima method with respective fractal indices given by $H \in[0.55,0.75]$ and $H \in[0.2,0.35]$. Fractal analysis is also linked with lesion detectability in mammogram textures. Actually, as observed in [18], it may be more difficult to detect lesion in dense tissues than in fatty tissues. This was mathematically proven in [30], using a-contrario model, for textures like isotropic fractional Brownian fields, showing that size and contrast of lesions are linearly linked in log-log plot with a slope depending on the $H$ index. This is illustrated in Figure 19, where we have added a spot with an increasing radius on two simulations of fractional Brownian fields of size $512 \times 512$ for $H=0.3$, corresponding to values of fatty tissues, and for $H=0.7$, corresponding to values of dense tissues. Contrarily to white noise images, obtained with independent identically distributed Gaussian variables on each pixel, in fractal images the more the radius increases, the less the spot is observable.

\section{Geometric construction}

We present here geometric construction for some fractional Brownian fields based on Chentsov's representation of random fields using a random measure and a par- 


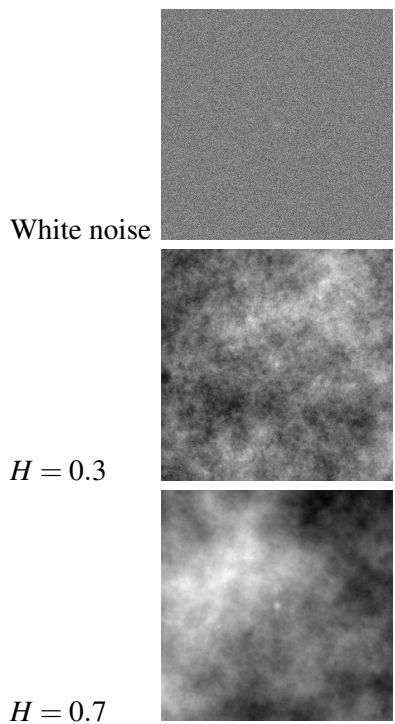

radius 5
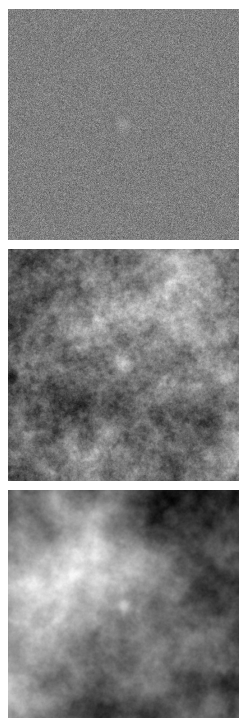

radius 10
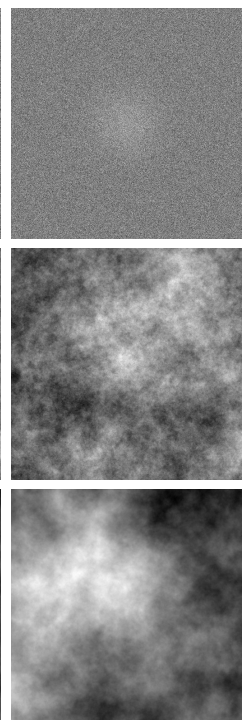

radius 50

Fig. 19 Examples of simulated spots with various sizes (radius 5, 10, and 50) but similar contrast in a white noise texture (top row) and in fractional Brownian fields $H=0.3$ and $H=0.7$ (bottom row).

ticular class of Borel sets indexed by points of $\mathbb{R}^{d}$. This is particularly interesting as it yields possible extensions, in particular beyond the Gaussian framework.

\subsection{Random measures}

A random measure will be defined as a stochastic process indexed by some Borel set. We still consider $(\Omega, \mathscr{A}, \mathbb{P})$ a probability space. Let $k \geq 1$ and $\mu$ be a $\sigma$-finite non-negative measure on $\left(\mathbb{R}^{k}, \mathscr{B}\left(\mathbb{R}^{k}\right)\right)$. Let set

$$
\mathscr{E}_{\mu}=\left\{A \in \mathscr{B}\left(\mathbb{R}^{k}\right) \text { such that } \mu(A)<+\infty\right\} .
$$

Definition 22. A random measure $M$ is a stochastic process $M=\left\{M(A) ; A \in \mathscr{E}_{\mu}\right\}$ satisfying

- For all $A \in \mathscr{E}_{\mu}, M(A)$ is a real random variable on $(\Omega, \mathscr{A})$;

- For $A_{1}, \ldots, A_{n} \in \mathscr{E}_{\mu}$ disjoint sets the random variables $M\left(A_{1}\right), \ldots, M\left(A_{n}\right)$ are independent;

- For $\left(A_{n}\right)_{n \in \mathbb{N}}$ disjoint sets such that $\cup_{n \in \mathbb{N}} A_{n} \in \mathscr{E}_{\mu}$, 


$$
M\left(\cup_{n \in \mathbb{N}} A_{n}\right)=\sum_{n \in \mathbb{N}} M\left(A_{n}\right) \text { a.s. }
$$

Let us emphasize that this definition does not ensure that almost surely $M$ is a measure. However some random measures may be almost surely measures.

Definition 23. A Poisson random measure $N$ with intensity $\mu$ is a random measure such that for any $A \in \mathscr{E}_{\mu}$, the random variable $N(A)$ follows a Poisson distribution of intensity $\mu(A)$ ie $N(A) \sim \mathscr{P}(\mu(A))$.

In this case $N$ is a random discrete measure given by $N=\sum_{i \in I} \delta_{T_{i}}$, where $\Phi=\left(T_{i}\right)_{i \in I}$ is a countable family of random variables with values in $\mathbb{R}^{k}$ called Poisson point process on $\mathbb{R}^{k}$ with intensity $\mu$ (see [37] for instance). For example, when $k=1$ and $\mu=\lambda \times$ Lebesgue for some $\lambda>0,(N([0, t]))_{t>0}$ is the classical Poisson process of intensity $\lambda$ and $\Phi$ corresponds to the jumps of the Poisson process.

Definition 24. A Gaussian random measure $W$ with intensity $\mu$ is a random measure such that for any $A \in \mathscr{E}_{\mu}$, the random variable $W(A)$ follows a normal distribution with mean 0 and variance $\mu(A)$, ie $W(A) \sim \mathscr{N}(0, \mu(A))$.

In this case, $W$ is not an a.s. measure. It is a centered Gaussian stochastic process (sometimes called set-indexed process, see [34] for instance) with covariance given by

$$
\operatorname{Cov}(W(A), W(B))=\mu(A \cap B)=\frac{1}{2}(\mu(A)+\mu(B)-\mu(A \Delta B)),
$$

for all $A, B \in \mathscr{E}_{\mu}$, with $A \Delta B=\left(A \cap B^{c}\right) \cup\left(B \cap A^{c}\right)$. Let us note that this is also the covariance function of any second order random measure $M$ satisfying $\operatorname{Var}(M(A))=$ $\mu(A)$ and so for $N$ a Poisson random measure of intensity $\mu$. For example, when $k=1$ and $\mu=\lambda \times$ Lebesgue for some $\lambda>0,(W([0, t]))_{t \geq 0}$ is the classical (nonstandard) Brownian motion with diffusion $\lambda$, up to continuity of sample paths. Conversely, considering a Brownian motion $\left(B_{t}\right)_{t \in \mathbb{R}}$ one can define a Gaussian random measure on $\mathbb{R}$ given by $W(A)=\int_{-\infty}^{+\infty} \mathbf{1}_{A}(t) d B_{t}$.

The link between Poisson and Gaussian measures is given by the central limit theorem. Actually, if $N^{(1)}, \ldots, N^{(n)}$ are independent Poisson random measures with the same intensity $\mu$, by superposition principle $\sum_{i=1}^{n} N^{(i)}$ is a Poisson random measure with intensity $n \times \mu$. By the central limit theorem we immediately deduce that for $A \in \mathscr{E}_{\mu}$

$$
\frac{1}{\sqrt{n}}\left(\sum_{i=1}^{n} N^{(i)}(A)-n \mu(A)\right) \underset{n \rightarrow+\infty}{\stackrel{d}{\rightarrow}} W(A) .
$$

More generally we have the following normal approximation for Poisson measures in high intensity.

Proposition 14. If $N_{\lambda}$ is a Poisson random measure with intensity $\lambda \times \mu$ and $W$ is a Gaussian random measure with the same intensity $\mu$, then

$$
\left(\lambda^{-1 / 2}\left(N_{\lambda}(A)-\lambda \mu(A)\right)\right)_{A \in \mathscr{E} \mu} \underset{\lambda \rightarrow+\infty}{\stackrel{f d d}{\longrightarrow}}(W(A))_{A \in \mathscr{E}_{\mu}}
$$




\subsection{Chentsov's representation: Lévy and Takenaka constructions}

Chentsov's type representation (see [56]) consists in constructing a random field $X$ with $M$ a random measure with intensity $\mu$ on $\mathbb{R}^{k}$ and $\mathscr{V}=\left\{V_{x} ; x \in \mathbb{R}^{d}\right\}$ a class of sets of $\mathscr{E}_{\mu}$ indexed by $\mathbb{R}^{d}$ for $d \geq 1$, by setting

$$
X_{x}=M\left(V_{x}\right), x \in \mathbb{R}^{d} .
$$

Then $X$ is called Chentsov random field associated with $M$ and $\mathscr{V}$. If $M$ is a second order random measure satisfying $\operatorname{Var}(M(A))=\mu(A)$ then $X$ is a second order random field with

$$
\forall x, y \in \mathbb{R}^{d}, \operatorname{Var}\left(X_{x}-X_{y}\right)=\mu\left(V_{x} \Delta V_{y}\right) .
$$

Then invariance properties of $X$ imply several relations on $\mu$ and $\mathscr{V}$. If $X$ has stationary increments then we must have $\mu\left(V_{x} \Delta V_{y}\right)=\mu\left(V_{x-y} \Delta V_{0}\right)$ for all $x, y \in \mathbb{R}^{d}$; If $X$ is isotropic and $X_{0}=0$ a.s. then $\mu\left(V_{R x}\right)=\mu\left(V_{x}\right)$, for all vectorial rotations $R$; Finally if $X$ is $H$-self-similar and $X_{0}=0$ a.s. then we obtain $\mu\left(V_{c x}\right)=c^{2 H} \mu\left(V_{x}\right)$, for all $c>0$. It follows that for $X$ to be isotropic, $H$ self-similar with stationary increments, we necessarily have $\mu\left(V_{x} \Delta V_{y}\right)=\mu\left(V_{x-y}\right)=c\|x-y\|^{2 H}, t, s \in \mathbb{R}^{d}$, for some constant $c>0$. This is only possible when $H \in(0,1 / 2]$. This comes from the fact that $V_{2 x} \subset\left(V_{2 x} \Delta V_{x}\right) \cup V_{x}$. Hence, by increments stationarity, $\mu\left(V_{2 x}\right) \leq$ $\mu\left(V_{x} \Delta V_{0}\right)+\mu\left(V_{x}\right) \leq 2 \mu\left(V_{x}\right)$ since $\mu\left(V_{0}\right)=\operatorname{Var}\left(X_{0}\right)=0$. By self-similarity we obtain that $2^{2 H} \mu\left(V_{x}\right) \leq 2 \mu\left(V_{x}\right)$ for all $x \in \mathbb{R}^{d}$, implying $H \leq 1 / 2$. We describe in the following the different constructions given by Lévy and Chentsov (1948 \& 1957) for $H=1 / 2$ and Takenaka (1987) for $H \in(0,1 / 2)$.

Proposition 15. Let $\mu$ and $\mathscr{V}$ be defined on $\left(\mathbb{R}^{d}, \mathscr{B}\left(\mathbb{R}^{d}\right)\right)$ by

- $\mu(d z)=\|z\|^{-d+1} d z$

- $\mathscr{V}=\left\{V_{x}, x \in \mathbb{R}^{d}\right\}$ with $V_{x}=B\left(\frac{x}{2}, \frac{\|x\|}{2}\right)=\left\{z \in \mathbb{R}^{d}:\left\|z-\frac{x}{2}\right\|<\frac{\|x\|}{2}\right\}$, the ball of diameter $[0, x]$, for all $x \in \mathbb{R}^{d}$.

Then, $\mu\left(V_{x} \Delta V_{y}\right)=\mu\left(V_{x-y}\right)=c_{d}\|x-y\|$, for all $x, y \in \mathbb{R}^{d}$.

Proof. For $x \in \mathbb{R}^{d}$, we may used polar coordinates to identify $V_{x}$ with $\left\{(r, \theta) \in \mathbb{R}_{+} \times S^{d-1}: 0<r<\theta \cdot x\right\}$ Then,

$$
\mu\left(V_{x}\right)=\int_{S^{d-1}} \int_{\mathbb{R}_{+}} 1_{\{r<\theta \cdot x\}} d r d \theta=\frac{1}{2} \int_{S^{d-1}}|\theta \cdot x| d \theta=\frac{c_{d}}{2}\|x\|,
$$

with $c_{d}=\int_{S^{d-1}}\left|e_{1} \cdot x\right| d \theta$. Moreover, for $y \neq x$,

$$
\begin{aligned}
\mu\left(V_{x} \cap V_{y}^{c}\right) & =\int_{S^{d-1}} \int_{\mathbb{R}_{+}} 1_{\{\theta \cdot y \leq r<\theta \cdot x\}} d r d \theta \\
& =\int_{0<\theta \cdot y<\theta \cdot x} \theta \cdot(x-y) d \theta+\int_{\theta \cdot y<0<\theta \cdot x} \theta \cdot x d \theta .
\end{aligned}
$$


Similarly, by a change of variables,

$$
\mu\left(V_{y} \cap V_{x}^{c}\right)=\int_{\theta \cdot y<\theta \cdot x<0}|\theta \cdot(x-y)| d \theta+\int_{\theta \cdot y<0<\theta \cdot x}(-\theta \cdot y) d \theta,
$$

so that

$$
\mu\left(V_{x} \Delta V_{y}\right)=\frac{1}{2} \int_{S^{d-1}}|\theta \cdot(x-y)| d \theta=\frac{c_{d}}{2}\|x-y\| .
$$

One can therefore check that the Chentsov random field associated with a Gaussian measure $W$ of intensity $\mu$ and $\mathscr{V}$, given in Proposition 15 is a (non-standard) Levy Chentsov field, or equivalently, a fractional Brownian field of index $H=1 / 2$. The construction for $H \in(0,1 / 2)$ has been given by Takenaka and relies on the following proposition.

Proposition 16. Let $H \in(0,1 / 2), \mu$ and $\mathscr{V}$ be defined on $\left(\mathbb{R}^{d+1}, \mathscr{B}\left(\mathbb{R}^{d+1}\right)\right)$ by

- $\mu_{H}(d z, d r)=r^{2 H-d-1} \mathbf{1}_{r>0} d z d r$ for $(z, r) \in \mathbb{R}^{d} \times \mathbb{R}$;

- $\mathscr{V}=\left\{V_{x}, x \in \mathbb{R}^{d}\right\}$ with $V_{x}=\mathscr{C}_{x} \Delta \mathscr{C}_{0}$ where $\mathscr{C}_{x}=\left\{(z, r) \in \mathbb{R}^{d} \times \mathbb{R}:\|z-x\| \leq r\right\}$, for all $x \in \mathbb{R}^{d}$.

Then, $\mu_{H}\left(V_{x} \Delta V_{y}\right)=\mu_{H}\left(V_{x-y}\right)=c_{H, d}\|x-y\|^{2 H}$, for all $x, y \in \mathbb{R}^{d}$.

Proof. Let $x \in \mathbb{R}^{d}$ with $x \neq 0$. Let us note that $\mu_{H}\left(\mathscr{C}_{x}\right)=+\infty$ but, integrating first with respect to $r$,

$$
\begin{aligned}
\mu_{H}\left(\mathscr{C}_{x} \cap \mathscr{C}_{0}^{c}\right) & =\frac{1}{d-2 H} \int_{\|z-x\|<\|z\|}\left(\|z-x\|^{2 H-d}-\|z\|^{2 H-d}\right) d z \\
& =c_{H, d}\|x\|^{2 H}=\mu_{H}\left(\mathscr{C}_{0} \cap \mathscr{C}_{x}^{c}\right)
\end{aligned}
$$

using translation invariance of Lebesgue's measure, where

$$
c_{H, d}=\frac{1}{d-2 H} \int_{\left\|z-e_{1}\right\|<\|z\|}\left(\left\|z-e_{1}\right\|^{2 H-d}-\|z\|^{2 H-d}\right) d z \in(0,+\infty) .
$$

Again by translation invariance of Lebesgue's measure, for $y \neq x$, we get $\mu_{H}\left(\mathscr{C}_{x} \Delta \mathscr{C}_{y}\right)=$ $\mu_{H}\left(\mathscr{C}_{x-y} \Delta \mathscr{C}_{0}\right)=c_{H, d}\|x-y\|^{2 H}$. The result follows once remarked that $V_{x} \Delta V_{y}=$ $\mathscr{C}_{x} \Delta \mathscr{C}_{y}$.

Of course, considering an associated Gaussian random measure we obtain the Chentsov's representation of fractional Brownian fields for $H \in(0,1 / 2)$. Let us remark that it also allows to define self-similar symmetric $\alpha$-stable fields considering an $\mathrm{S} \alpha \mathrm{S}$ random measure (see [56]) but we leave our second order framework in this way! However, considering instead a Poisson random measure, we can define a Poisson analogous of fractional Brownian fields when $H \in(0,1 / 2)$. 


\subsection{Fractional Poisson fields}

When $N_{\lambda, H}$ is a Poisson random measure on $\mathbb{R}^{d} \times \mathbb{R}$ with intensity $\lambda \times \mu_{H}$ for $\lambda>0$, and $\mu_{H}$ given by Proposition 16, we can define a Chentsov's field by

$$
N_{\lambda, H}\left(\mathscr{C}_{x} \Delta \mathscr{C}_{0}\right)=N_{\lambda, H}\left(\mathscr{C}_{x} \cap \mathscr{C}_{0}^{c}\right)+N_{\lambda, H}\left(\mathscr{C}_{x}^{c} \cap \mathscr{C}_{0}\right), \forall x \in \mathbb{R}^{d}
$$

However, since $N_{\lambda, H}\left(\mathscr{C}_{x} \Delta \mathscr{C}_{0}\right)$ is a Poisson random variable of parameter $\lambda \mu_{H}\left(\mathscr{C}_{x} \Delta \mathscr{C}_{0}\right)$, this field is not a centered. But remarking that $\mu_{H}\left(\mathscr{C}_{x} \cap \mathscr{C}_{0}^{c}\right)=\mu_{H}\left(\mathscr{C}_{x}^{c} \cap \mathscr{C}_{0}\right)$, we may define the centered fractional Poisson field on $\mathbb{R}^{d}$ by

$$
F_{\lambda, H}(x)=N_{\lambda, H}\left(\mathscr{C}_{x} \cap \mathscr{C}_{0}^{c}\right)-N_{\lambda, H}\left(\mathscr{C}_{x}^{c} \cap \mathscr{C}_{0}\right), \forall x \in \mathbb{R}^{d}
$$

Actually, $F_{\lambda, H}$ may also be defined as the stochastic integral with respect to the Poisson random measure $N_{\lambda, H}$ as

$$
\begin{aligned}
F_{\lambda, H}(x) & =\int_{\mathbb{R}^{d} \times \mathbb{R}}\left(\mathbf{1}_{\mathscr{C}_{x} \cap \mathscr{C}_{0}^{c}}(z, r)-\mathbf{1}_{\mathscr{C}_{x}^{c} \cap \mathscr{C}_{0}}(z, r)\right) N_{\lambda, H}(d z, d r) \\
& =\int_{\mathbb{R}^{d} \times \mathbb{R}}\left(\mathbf{1}_{B(z, r)}(x)-\mathbf{1}_{B(z, r)}(0)\right) N_{\lambda, H}(d z, d r)
\end{aligned}
$$

Heuristically, we can throw centers and radius with respect to a Poisson point process on $\mathbb{R}^{d} \times \mathbb{R}$ of intensity $\lambda \times \mu_{H}$, meaning that centers are thrown uniformly in $\mathbb{R}^{d}$ with intensity $\lambda$ and independently marked with a radius. Then $F_{\lambda, H}(x)$ will count the number of balls falling on $x$ minus the number of balls falling on 0 . It is close to a shot noise random field obtained as the sum of randomly dilated and translated contributions. We refer to [10] and [9] for more details. Then $\left(F_{\lambda, H}(x)\right)_{x \in \mathbb{R}^{d}}$ is centered, with stationary increments, isotropic with covariance

$$
\operatorname{Cov}\left(F_{\lambda, H}(x), F_{\lambda, H}(y)\right)=\frac{\lambda c_{H, d}}{2}\left(\|x\|^{2 H}+\|y\|^{2 H}-\|x-y\|^{2 H}\right) .
$$

This field is not self-similar but

$$
\left(F_{\lambda, H}(c x)\right)_{x \in \mathbb{R}^{d}} \stackrel{f d d}{=}\left(F_{\lambda c^{2 H}, H}(x)\right)_{x \in \mathbb{R}^{d}}, \forall c>0 .
$$

Moreover, according to normal approximation of Poisson measures for high intensity we can prove that $\left(\lambda^{-1 / 2} F_{\lambda, H}(x)\right)_{x \in \mathbb{R}^{d}} \underset{\lambda \rightarrow+\infty}{\stackrel{f d d}{\longrightarrow}}\left(\sqrt{C_{H, d}} B_{H}(x)\right)_{x \in \mathbb{R}^{d}}$. This is illustrated in Figure 20. Note that sharp contours of fractional Poisson fields disappear in the asymptotic Gaussian limits, that are Hölder continuous. Another interesting property of this field is that its distribution is also preserved along lines (and more generally along affine subspaces). More precisely, for $x_{0} \in \mathbb{R}^{d}$ and $\theta \in S^{d-1}$, defining the line process $L_{x_{0}, \theta}\left(F_{\lambda, H}\right)=\left(F_{\lambda, H}\left(x_{0}+t \theta\right)\right)_{t \in \mathbb{R}}$, computing characteristic functions we can prove that $\left(L_{x_{0}, \theta}(t)-L_{x_{0}, \theta}(0)\right)_{t \in \mathbb{R}}$ has the same distribution than a fractional Poisson process (defined for $d=1$ ), with the same $H$ index and intensity given by $c_{H, d} \lambda$ where $c_{H, d}=\int_{\mathbb{R}^{d-1}}\left(1-\|y\|^{2}\right)^{1 / 2-H} \mathbf{1}_{\|y\| \leq 1} d y$ (see [10]). 

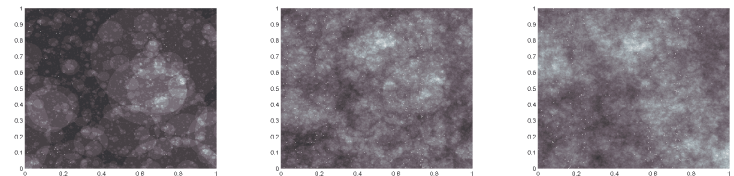

Fig. 20 Convergence of a fractional Poisson field to a fractional Brownian field as the intensity increases.

Hence, we can also use estimation based on variograms to build estimators of $H$. Consistency has been proven in [9]. Figure 21 presents sample paths comparison between Poisson and Gaussian cases. To conclude, let us note that, contrarily to the Gaussian case, one can prove that the increments $\mathbb{E}\left(\left|F_{\lambda, H}(x)-F_{\lambda, H}(x)\right|^{q}\right)$ behave like $\|x-y\|^{2 H}$ for any $q \geq 2$ as $\|x-y\| \rightarrow 0$. Such a feature still holds allowing some interactions for the radii as done in [54].
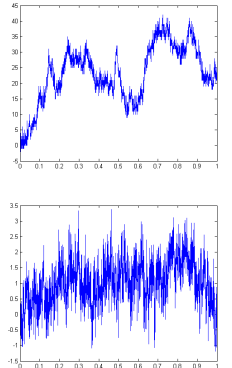

$H=0.1$
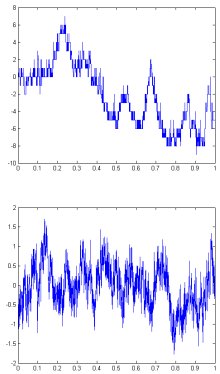

$H=0.2$
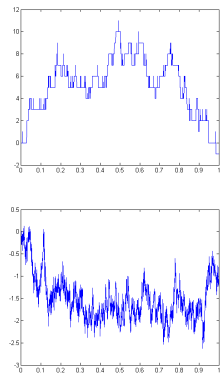

$H=0.3$
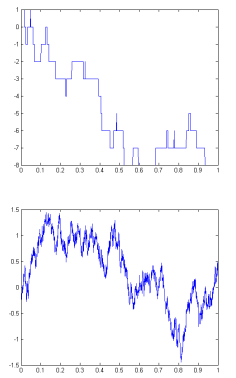

$H=0.4$

Fig. 21 Sample paths of fractional Poisson process (top) vs fractional Brownian motion (bottom)

Acknowledgements I would like to warmly thanks all my co-authors for the different works partially presented here, especially Clément Chesseboeuf and Olivier Durieu for their careful reading.

\section{References}

1. R. J. Adler. The Geometry of Random Field. John Wiley \& Sons, 1981.

2. D. Allard, R. Senoussi, and E. Porcu. Anisotropy models for spatial data. Math. Geosci., 48(3):305-328, 2016.

3. A. Ayache and F. Roueff. A Fourier formulation of the Frostman criterion for random graphs and its applications to wavelet series. Appl. Comput. Harmon. Anal., 14:75-82, 2003.

4. A. Benassi, S. Cohen, and J. Istas. Local self-similarity and the Hausdorff dimension. C.R. Acad Sci. Paris Ser.I, 336(3):267-272, 2003. 
5. C. L. Benhamou, S. Poupon, E. Lespessailles, S. Loiseau, R. Jennane, V. Siroux, W. Ohley, and L. Pothuaud. Fractal Analysis of radiographic Trabecular Bone Texture and Bone Mineral Density: Two Complementary Parameters Related to Osteoporotic Fractures. Journal of bone and mineral research, 16(4):697-704, 2001.

6. C. Berzin, A. Latour, and J. R. León. Inference on the Hurst parameter and the variance of diffusions driven by fractional Brownian motion, volume 216 of Lecture Notes in Statistics. Springer, Cham, 2014. With a foreword by Aline Bonami.

7. H. Biermé, C.L. Benhamou, and F. Richard. Parametric estimation for gaussian operator scaling random fields and anisotropy analysis of bone radiograph textures. In K. Pohl, editor, Proc. of the International Conference on Medical Image Computing and Computer Assisted Intervention (MICCAI'09), Workshop on Probabilistic Models for Medical Imaging, pages 13-24, London, UK, september 2009.

8. H. Biermé, A. Bonami, and J. R. León. Central limit theorems and quadratic variations in terms of spectral density. Electronic Journal of Probability, 16(3):362-395, 2011.

9. H. Biermé, Y. Demichel, and A. Estrade. Fractional Poisson field and fractional Brownian field: why are they resembling but different? Electron. Commun. Probab., 18:11-13, 2013.

10. H. Biermé, A. Estrade, and I. Kaj. Self-similar random fields and rescaled random balls models. J. Theoret. Probab., 23(4):1110-1141, 2010.

11. H. Biermé and C. Lacaux. Fast and exact synthesis of some operator scaling gaussian random fields, 2017. Preprint.

12. H. Biermé, M. M. Meerschaert, and H. P. Scheffler. Operator scaling stable random fields. Stoch. Proc. Appl., 117(3):312-332, 2007.

13. H. Biermé, L. Moisan, and F. Richard. A turning-band method for the simulation of anisotropic fractional brownian fields. J. Comput. Graph. Statist, 24(3), 2015.

14. H. Biermé and F. Richard. Statistical tests of anisotropy for fractional brownian textures. application to full-field digital mammography. J. Math. Imaging Vis., 36(3):227-240, 2010.

15. M. Bilodeau and D. Brenner. Theory of multivariate statistics. Springer Texts in Statistics. Springer-Verlag, New York, 1999.

16. A. Bonami and A. Estrade. Anisotropic analysis of some Gaussian models. J. Fourier Anal. Appl., 9(3):215-236, 2003.

17. P. Breuer and P. Major. Central limit theorems for nonlinear functionals of Gaussian fields. $J$. Multivariate Anal., 13(3):425-441, 1983.

18. A. Burgess, F. Jacobson, and P. Judy. Human observer detection experiments with mammograms and power-law noise. Med. Phys., 28(4):419-437, 2001.

19. C. Caldwell, S. Stapleton, D. Holdsworth, et al. On the statistical nature of characterisation of mammographic parenchymal patterns by fractal dimension. Phys. Med. Biol., 35(2):235-247, 1990.

20. C. B. Caldwell, J. Rosson, J. Surowiak, and T Hearn. Use of fractal dimension to characterize the structure of cancellous bone in radiographs of the proximal femur. In Fractals in Biology and Medicine, pages 300-306, 1994.

21. G. Chan. An effective method for simulating Gaussian random fields. In Proceedings of the statistical Computing section, pages 133-138, www.stat.uiowa.edu/grchan/, 1999. Amerir. Statist.

22. S. Cohen and J. Istas. Fractional fields and applications, volume 73 of Mathématiques \& Applications (Berlin) [Mathematics \& Applications]. Springer, Heidelberg, 2013. With a foreword by Stéphane Jaffard.

23. P. F. Craigmile. Simulating a class of stationary Gaussian processes using the Davies-Harte algorithm, with application to long memory processes. J. Time Ser. Anal., 24(5):505-511, 2003.

24. Robert Dalang, Davar Khoshnevisan, Carl Mueller, David Nualart, and Yimin Xiao. A minicourse on stochastic partial differential equations, volume 1962 of Lecture Notes in Mathematics. Springer-Verlag, Berlin, 2009. Held at the University of Utah, Salt Lake City, UT, May 8-19, 2006, Edited by Khoshnevisan and Firas Rassoul-Agha.

25. S. Davies and P. Hall. Fractal analysis of surface roughness by using spatial data. J. R. Stat. Soc. Ser. B, 61:3-37, 1999. 
26. C. R. Dietrich and G. N. Newsam. Fast and exact simulation of stationary gaussian processes through circulant embedding of the covariance matrix. SIAM J. Sci. Comput., 18(4):10881107, 1997.

27. K. J. Falconer. Fractal Geometry. John Wiley \& Sons, 1990.

28. W. Feller. An introduction to probability theory and its applications. Vol. II. Second edition. John Wiley \& Sons, Inc., New York-London-Sydney, 1971.

29. T. Gneiting, H. Sevciková, D.B. Percivala, M. Schlather, and Y. Jianga. Fast and exact simulation of large gaussian lattice systems in $\mathbb{R}^{2}$ : Exploring the limits. Journal of Computational and Graphical Statistics, 15:483-501, 1996.

30. B. Grosjean and L. Moisan. A-contrario detectability of spots in textured backgrounds. $J$. Math. Imaging Vision, 33(3):313-337, 2009.

31. R. Harba, G. Jacquet, R. Jennane, T. Loussot, C. L. Benhamou, E. Lespessailles, and D. Tourlire. Determination of fractal scales on trabecular bone x-ray images. Fractals, 2(3):451-456, 1994.

32. K. Harrar, R. Jennane, K. Zaouchi, T. Janvier, H. Toumi, and E. Lespessailles. Oriented fractal analysis for improved bone microarchitecture characterization. Biomed. Signal Proc. and Control, 39:474-485, 2018.

33. J. Heine and R. Velthuizen. Spectral analysis of full field digital mammography data. Med. Phys., 29(5):647-661, 2002.

34. E. Herbin and E. Merzbach. The set-indexed Lévy process: stationarity, Markov and sample paths properties. Stochastic Process. Appl., 123(5):1638-1670, 2013.

35. J. Istas. On fractional stable fields indexed by metric spaces. Elect. Comm. in Probab., 11:242$251,2006$.

36. J. Istas and G. Lang. Quadratic variations and estimation of the local Hlder index of a Gaussian process. Ann. Inst. Henri Poincaré, Prob. Stat., 33(4):407-436, 1997.

37. O. Kallenberg. Foundations of modern probability. Probability and its Applications (New York). Springer-Verlag, New York, 1997.

38. L. M. Kaplan and C. C. J. Kuo. An Improved Method for 2-d Self-Similar Image Synthesis. IEEE Trans. Image Process., 5(5):754-761, 1996.

39. I. Karatzas and E. Shreve. Brownian Motion and Stochastic Calculus. Springer-Verlag, 1998.

40. P. Kesterner, J.M. Lina, P. Saint-Jean, and A. Arneodo. Waveled-based multifractal formalism to assist in diagnosis in digitized mammograms. Image Anal. Stereol., 20:169-174, 2001.

41. A. N. Kolmogorov. The local structure of turbulence in an incompressible viscous fluid for very large reynolds number. Dokl. Akad. Nauk SSSR, 30:301-305, 1941.

42. R. Leipus, A. Philippe, D. Puplinskaite, and D. Surgailis. Aggregation and long memory: recent developments. J. Indian Statist. Assoc., 52(1):81-111, 2014.

43. E. Lespessailles, C. Gadois, I. Kousignian, Fardellone P. Neveu, J.P., S. Kolta, C. Roux, J.P. Do-Huu, and C.L. Benhamou. Clinical interest of bone texture analysis in osteoporosis: a case control multicenter study. Osteoporos. Int., 19:1019-1028, 2008.

44. Y. Li, W. Wang, and Y. Xiao. Exact moduli of continuity for operator-scaling Gaussian random fields. Bernoulli, 21(2):930-956, 2015.

45. G. Lindgren. Stationary stochastic processes. Chapman \& Hall/CRC Texts in Statistical Science Series. CRC Press, Boca Raton, FL, 2013. Theory and applications.

46. R. Lopes and N. Betrouni. Fractal and multifractal analysis: a review. Med. Image Anal., 13:634-649, 2009.

47. B. B. Mandelbrot and J. Van Ness. Fractional Brownian motion, fractionnal noises and applications. Siam Review, 10:422-437, 1968.

48. G. Matheron. The intrinsic random functions and their application. Adv. Appl. Prob., 5:439$468,1973$.

49. I. Molchanov and K. Ralchenko. A generalisation of the fractional Brownian field based on non-Euclidean norms. J. Math. Anal. Appl., 430(1):262-278, 2015.

50. G. Peccati and C. Tudor. Gaussian limits for vector-valued multiple stochastic integrals. Séminaire de Probabilités, XXXVIII:247-262, 2004. 
51. E. Perrin, R. Harba, R. Jennane, and I. Iribarren. Fast and exact synthesis for 1-D fractional Brownian motion a nd fractional gaussian noises. IEEE Signal Processing Letters, 9(11):382384, 2002.

52. V. Pilipauskaitė and D. Surgailis. Scaling transition for nonlinear random fields with longrange dependence. Stochastic Process. Appl., 127(8):2751-2779, 2017.

53. C. E. Powell. Generating realisations of stationary gaussian random fields by circulant embedding, 2014. Technical report.

54. N. Privault. Poisson sphere counting processes with random radii. ESAIM Probab. Stat., 20:417-431, 2016.

55. W. Rudin. Real and complex Analysis. McGraw-Hill, 1986.

56. G. Samorodnitsky and M. S. Taqqu. Stable non-Gaussian random processes. Stochastic Modeling. Chapman \& Hall, New York, 1994. Stochastic models with infinite variance.

57. M. L. Stein. Fast and exact simulation of fractional Brownian surfaces. J. Comput. Graph. Statist., 11(3):587-599, 2002.

58. A. W. van der Vaart. Asymptotic statistics, volume 3 of Cambridge Series in Statistical and Probabilistic Mathematics. Cambridge University Press, Cambridge, 1998. 